\title{
Material aspects of triboelectric energy generation and sensors
}

\author{
Dong Wook Kim¹, Ju Hyun Lee², Jin Kon Kim² and Unyong Jeong (1)
}

\begin{abstract}
The triboelectric nanogenerator (TENG) is a new type of energy generator first demonstrated in 2012. TENGs have shown potential as power sources for electronic devices and as sensors for detecting mechanical and chemical stimuli. To date, studies on TENGs have focused primarily on optimizing the systems and circuit designs or exploring possible applications. Even though triboelectricity is highly related to the material properties, studies on materials and material designs have been relatively less investigated. This review article introduces recent progress in TENGs, by focusing on materials and material designs to improve the electrical output and sensing performance. This article discusses the current technological issues and the future challenges in materials for TENG.
\end{abstract}

\section{Introduction}

During the last decade, triboelectric nanogenerators (TENGs) have been verified to effectively convert daily mechanical energy into useful electric power. Not only natural energy such as rain or wind but also everyday body motion such as hand touching or walking can serve as a mechanical energy source for triboelectrification ${ }^{1-3}$. In TENG devices, charges are separated on the contact surfaces, and an electrical potential is generated between the surfaces. The alternating potential resulting from the dynamic mechanical motions can be stored in a storage unit or can be used for powering electric devices ${ }^{4}$. In addition, the potential profiles or the current profiles of the TENG can also be used as sensing signals to monitor the mechanical or chemical stimuli exerted on TENGs ${ }^{5}$.

To date, system design and circuit design have been extensively studied to improve the output performance of TENGs. Four different modes (contact-separation mode ${ }^{6-8}$, sliding mode ${ }^{9,10}$, single-electrode mode ${ }^{11,12}$, and freestanding mode ${ }^{13,14}$ ) have been established, and a few

\footnotetext{
Correspondence: Unyong Jeong (ujeong@postech.ac.kr)

${ }^{1}$ Department of Materials Science and Engineering, Pohang University of

Science and Technology (POSTECH), 77, Cheongam-Ro, Nam-Gu, Pohang, Korea

${ }^{2}$ Department of Chemical Engineering, Pohang University of Science and

Technology (POSTECH), 77, Cheongam-Ro, Nam-Gu, Pohang, Korea

These authors contributed equally: Dong Wook Kim, Ju Hyun Lee
}

device structures by using elastic spacers ${ }^{15-18}$ or arcshape substrates ${ }^{19}$ have been suggested. Ideal cyclic device operation and the corresponding circuit design have been proposed theoretically and tested experimentally ${ }^{20,21}$. Analog circuits have been developed to analyze the irregular TENG profiles and to minimize the power loss caused by the signal irregularity ${ }^{22}$. Relatively, material design to improve the TENG performance has not been investigated thoroughly. Microscopic understanding of the electrification mechanism is not well established, and the materials used for TENGs are limited to a few species $^{23-25}$. Most studies on materials have focused on the morphological optimization of the contact surfaces ${ }^{3,26,27}$.

In this review article, we introduce recent progress in the material design for TENGs. The material aspects of enhancing the electric power output are discussed first, and the material designs for sensor applications are discussed next. This review ends with future challenges and suggestions for materials for TENG devices.

\section{Material approaches to enhance the triboelectric power}

In this section, we introduce the progress in materials for four representative component layers in TENGs: the charge-generating layer, the charge-trapping layer, the charge-collecting layer, and the charge-storage layer 


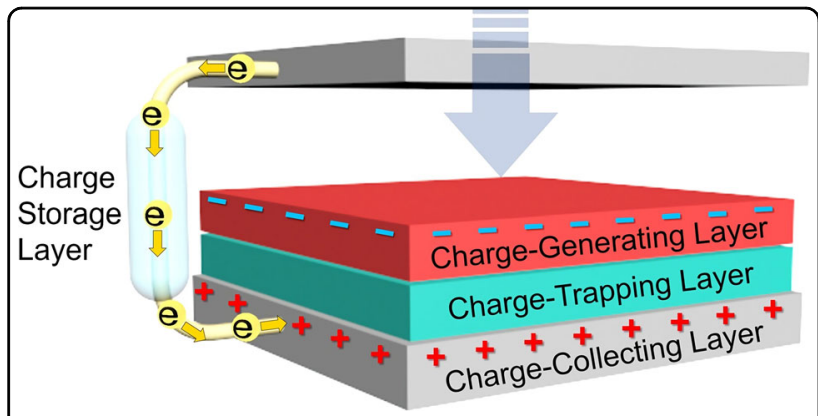

Fig. 1 Representative components of a triboelectric nanogenerator (TENG) affecting triboelectric power generation. The chargegenerating layer (red), the charge-trapping layer (green), the chargecollecting layer (gray), and the charge-storage layer (white) can affect power generation in TENG.

(Fig. 1). The approaches for designing the materials used in those layers are discussed, and future directions are suggested.

\section{Charge-generating layer}

Because the performance of TENGs is proportional to the charge density of the contact surface ${ }^{28}$, increasing the charge generation has been the main strategy to improve the output electric power. The square of the charge density is the main parameter used to quantify a TENG's performance as a material figure of merit ${ }^{29}$. Several approaches from the materials perspective have been reported $^{30-57}$. The approaches include increasing the contact area by fabricating microscale or nanoscale surface structures, surface functionalization to increase the surface charge density or to facilitate triboelectric charge transfer, modification of the surface property by electrical poling or doping, and creation of new materials by molecular synthesis or nanocomposite formation.

Increasing the contact surface area is a straightforward route to improve the total number of generated charges. When the two surfaces make contact by mechanical force, the actual contact area is much smaller than the surface areas of the two surfaces ${ }^{37}$. Fabricating microstructures on the surface has been widely investigated as a means to increase the contact area. Various methods to fabricate the microstructures have been reported, including forceassembled colloidal arrays ${ }^{30}$, soft lithography ${ }^{31}$, anodic aluminum oxide $(\mathrm{AAO})^{32}$, block-copolymer assembly ${ }^{33}$, and surface nanomaterial fabrication ${ }^{34,35}$. The microstructure on the hydrophobic surface not only improves the output performance of a TENG but also improves the sustainability of the performance in humid environments. These approaches are reviewed in several reports ${ }^{24,30}$. To date, most studies on the microstructured surfaces have focused on the increase in surface area ${ }^{24,30-36}$. Although triboelectrification is based on charging during contact separation or surface friction, adhesion between the contact materials has rarely been investigated. Recently, Lee et al. reported that increasing the adhesion energy can enhance the surface charge generation ${ }^{37}$ (Fig. 2a). They formed poly(dimethylsiloxane) (PDMS) micropillars on a PDMS substrate and used a PDMS thin film to cover the top of the pillars. The large elastic deformation of the PDMS thin film during contact and separation stored the applied mechanical energy; therefore, the charge generation on the adhesive surfaces was more effective than that on nonadhesive surfaces. To clearly understand the enhancement of charge generation by microstructure formation, the relationship between the deformation dynamics of the microstructures and the charge distribution near the microstructures should also be investigated. These studies need to control the viscoelasticity and surface adhesion of the microstructures. Recently, liquid-solid contact electrification has been studied on microstructured surfaces ${ }^{38,39}$. Combining the liquid-solid contacts and the solid-solid contacts may provide a good strategy to separate the effects of the increase in the surface area from the effect of the microstructure deformation dynamics. In addition, liquid-liquid contact electrification between two pure liquids can generate triboelectricity ${ }^{40}$. The droplets falling through a freestanding liquid membrane can provide continuous power generation (peak power of $137.4 \mathrm{nW}$ ) and can be applied to many targets, including raindrops, microfluidics, and irrigation currents.

A large difference in surface potentials between two contact surfaces results in a large triboelectric output ${ }^{41}$; thus, functionalization of the material surface is one of the key approaches to increasing the charge density of TENGs. Surface modification through ion doping, radical injection, and plasma treatment can have a significant impact on the charge generation ${ }^{42-45}$. When a fluorinated ethylene propylene (FEP) substrate was exposed to negatively ionized air, the electron transfer from an aluminum to the FEP surface was greatly increased ${ }^{42}$ (Fig. 2b). After injecting oxygen radicals into the PDMS surface, sodium hydroxide treatment converted the nonpolar $\mathrm{Si}-\mathrm{CH}_{3}$ bonds to polar $\mathrm{Si}-\mathrm{O}$ bonds, resulting in a 15 -fold enhancement in power over the untreated $\mathrm{PDMS}^{43}$. Fluorinated PDMS and fluorinated poly(ethylene terephthalate) (PET) substrates prepared by tetrafluoromethane $\left(\mathrm{CF}_{4}\right)$ plasma treatment considerably improved the energy density ${ }^{44,45}$. Self-assembled monolayers with electron-donating and electron-withdrawing functional end groups effectively changed the surface dipoles of the contact layer ${ }^{46,47}$, hence significantly enhancing or reducing the triboelectric output.

Changing the intrinsic material properties (dielectric constant, polarity, work function, etc.) has been pursued through nanocomposite formation, electrical poling, 
a

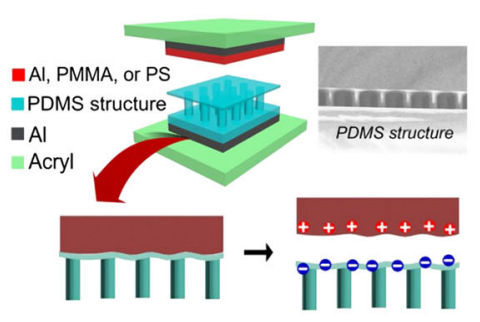

b

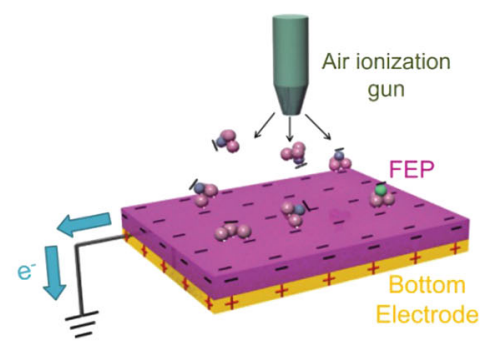

d
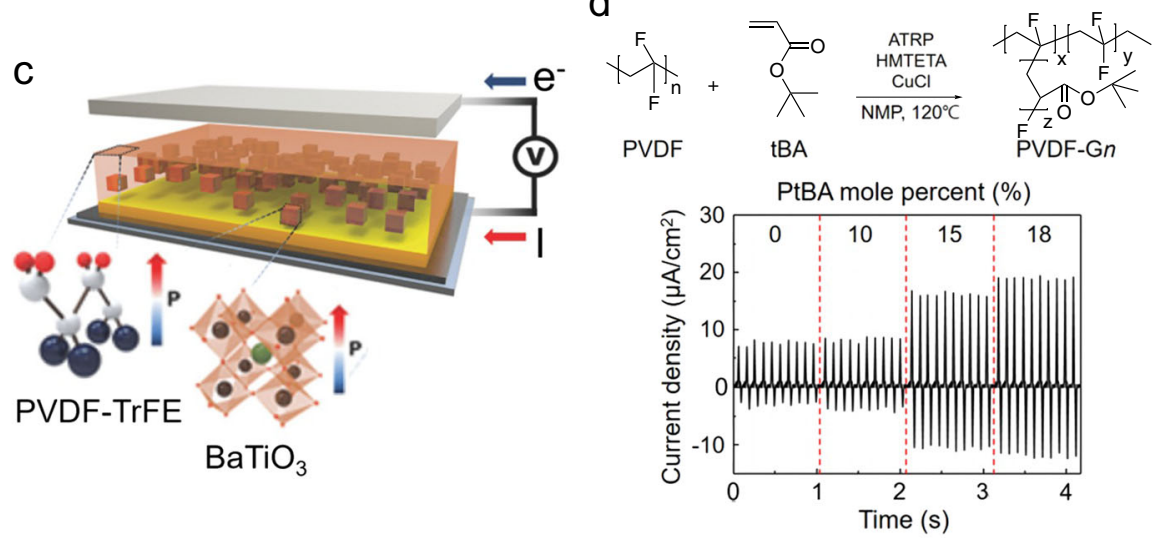

e

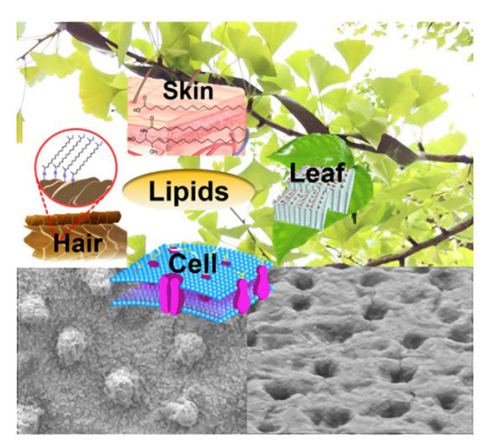

f

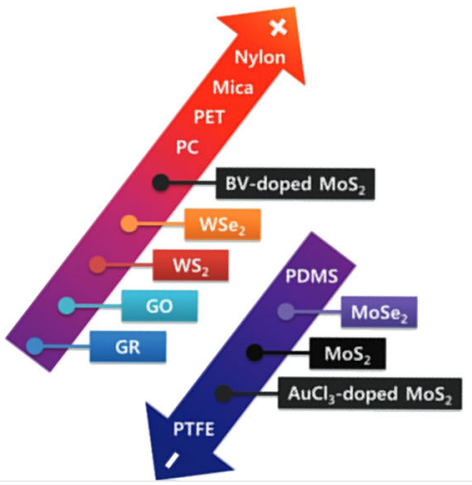

Fig. 2 Utilization of the charge-generating layer for power enhancement of TENGs. a Increased triboelectrification by enhancing the conformal contact and adhesion energy with a film-covered pillar structure (modified from ref. ${ }^{37}$, copyright permission from Elsevier). b Maximizing surface charge density achieved by ionized-air injection (modified from ref. ${ }^{42}$, copyright permission from Wiley-VCH). c Boosting power generation via control of ferroelectric polarization and dielectric properties (modified from ref. ${ }^{50}$, copyright permission from Wiley-VCH). $\mathbf{d}$ Enhanced current density output with the synthesis of grafted copolymers for dielectric constant control (modified from ref. ${ }^{53}$, copyright permission from American Association for the Advancement of Science). e Triboelectricity of regenerative natural substances covered with lipid layers (modified from ref. ${ }^{54}$, copyright permission from Wiley-VCH). $\mathbf{f}$ Triboelectric series of 2D layered materials (modified from ref. ${ }^{57}$, copyright permission from Wiley-VCH).

chemical doping, and material synthesis. Poly(vinylidene fluoride-co-trifluoroethylene) (PVDF-TrFE), which is a well-known ferroelectric polymer, can be polarized by electrical poling ${ }^{48}$. Lee et al. demonstrated that after electrical poling, PVDF-TrFE was ranked higher in the positive triboelectric series than human skin ${ }^{49}$. In an effort to improve charge generation, nanocomposite materials have been studied extensively. Electrical poling of a nanocomposite consisting of PVDF-TrFE and high- $k$ barium titanate $\left(\mathrm{BaTiO}_{3}\right)$ nanoparticles resulted in an 18fold increase in charge generation compared with that of pure PVDF-TrFE ${ }^{50}$ (Fig. 2c). Nanocomposites of poly (vinylidene fluoride) (PVDF) and zinc oxide ( $\mathrm{ZnO}$ ) nanorods ${ }^{58}$ or zinc stannate $\left(\mathrm{ZnSnO}_{3}\right)$ nanocubes ${ }^{59}$ exhibited 60 and $200 \%$ higher instantaneous output currents than the bare PVDF film-based TENGs, respectively. These significant output increases were attributed not only to the enhancement in $\beta$-phase content in a ferroelectric PVDF but also to the enhancement in surface roughness and hydrophobicity. When PVDF was doped with an electrolyte with an asymmetric ion pair, the triboelectrification property dramatically changed. 
Depending on the type of doping ions, PVDF varied from the most negative material to the most positive material ${ }^{51}$. $\mathrm{Kim}$ et al. reported that deposition of gold $\mathrm{(Au})$ nanoparticles coated with positively charged 4-(dimethylamino)pyridine (DMAP) on the PDMS contact surface enhanced the charge density by a factor of $2^{52}$. This study revealed that the electron transfer from the $\mathrm{Au}$ nanoparticles to the dielectrics became easier because the effective work function of the $\mathrm{Au}$ nanoparticle was decreased by the permanent dipole induced at the DMAP-Au interface. A material with a high dielectric constant can be a good candidate for high-density charge generation. Grafting of poly(tert-butyl acrylate) (PtBA) in the PVDF polymer chain remarkably increased the charge density in proportion to the mole fraction of PtBA in the graft copolymer ${ }^{53}$ (Fig. 2d).

Since Johan Carl Wilcke published the first triboelectric series in 1757, little change has been made in the material species. Recently, several new materials have been added to the triboelectric series. Kim et al. reported that lipid layers on the surface of regenerative natural substances (skin, hair, leaves, and cells) are responsible for the highly positive triboelectricity $^{54}$ (Fig. 2e). Similar to this study, other studies have utilized leaves and petals as the charge-generating layers of TENGs ${ }^{55,56}$. The charge density of a lipid-based TENG was four times higher than the charge density of a nylon 6-based TENG. These naturally regenerative materials open up the possibility of energy harvesting without damaging or changing the natural environment. Kim et al. also revealed that commercially available synthetic lipids have similar triboelectric characteristics regardless of the functional groups in the lipid molecules ${ }^{54}$. Unfortunately, the reason why the lipids have such a high positive charge density is not yet understood. In-depth studies on the charge distribution of a single molecule, the effect of the self-assembled structure of the lipids, and the effect of crystal deformation under mechanical contacts are needed. Two-dimensional (2D) materials $\left(\mathrm{MoS}_{2}, \mathrm{MoSe}_{2}, \mathrm{WS}_{2}\right.$, $\mathrm{WSe}_{2}$, graphene, graphene oxide, etc.) are relatively new materials and have been tested for TENGs. Seol et al. investigated the triboelectric behavior of various 2D materials and placed their ranks in the triboelectric series ${ }^{57}$ (Fig. 2f).

\section{Charge-trapping layer}

The triboelectric surface potential at one surface induces the opposite electrostatic potential at the interface with the counter electrode. The surface charges can be drifted by the electric field and combined with the induced opposite charges. This charge combination interferes with surface charge accumulation and causes a sharp decrease in the triboelectric potential. Although it is important to block the charge combination, studies of the insulating interlayer and charge-trapping layer are relatively new. This section introduces the recent advances in materials for blocking the charge combination.

In polymer insulators, charge traps are formed by physical defects such as amorphous free volume, crosslinking points, and imperfections in the crystal lattice and chemical defects such as dangling bonds and functional groups in the polymer chains ${ }^{60,61}$. Previous studies have reported that polymers containing aromatic rings in their chains, such as in polystyrene (PS) and polyimide (PI), have many trapping sites due to the nonuniform energy levels along their main chains ${ }^{62}$. The addition of aromatic polymer films between the PVDF layer and the collector electrode trapped triboelectric charges and increased the triboelectric output by 7-9 times compared with the device without the interlayer ${ }^{63,64}$ (Fig. 3a). PDMS has been found to have deeper charge traps and a larger trap density than the aromatic polymers (Fig. 3b). Adding a PDMS thin layer below the charge-generating layer increased the power density of TENGs by 178 times. In addition, the high stretchability of the PDMS layer enabled the fabrication of stretchable TENGs ${ }^{65}$. Various metal oxide thin films have been reported to block the charge combination due to their high permittivity, a large number of oxygen vacancies, and the high interface density of states. Adding a titanium oxide $\left(\mathrm{TiO}_{\mathrm{X}}\right)$ layer maintained the high surface charge density by suppressing the charge combination; thus, a significant enhancement in TENG performance was obtained ${ }^{66}$. A TENG with an indium zinc oxide (IZO) interlayer was also reported to provide a large interface density of states and to function as a charge reservoir ${ }^{67}$.

Nanomaterials have larger surface-to-volume ratios than thin-film interlayers and are likely to provide more charge trapping. It has been shown that monolayer $\mathrm{MoS}_{2}$ acts as a triboelectric electron acceptor and restricts the charge combination so that polymer composites containing monolayer $\mathrm{MoS}_{2}$ nanosheets improved the power density of TENGs $^{68}$. In a similar mechanism, composites of reduced graphene oxide (rGO) nanosheets effectively trapped electrons and resulted in a large output enhancement ${ }^{69}$. Black phosphorous (BP), decorated with hydrophobic cellulose oleoyl ester nanoparticles, served as a synergistic electrontrapping additive. These printable and coatable composites introduce the possibility of manufacturing high-performance textile TENGs ${ }^{70}$ (Fig. 3c). The large interface between the conductive fillers and the polymer matrix in nanocomposites can act as charge-trapping sites. In PVDF nanofibers, the addition of $\mathrm{Ag}$ nanowires ( $\mathrm{Ag} \mathrm{NWs}$ ) promoted the formation of a $\beta$-crystalline phase in the PVDF chains and trapped the electrostatically induced charges at the metal-dielectric interfaces. The synergistic effect increased the output performance of TENGs ${ }^{71}$ (Fig. 3d). Incorporating nanocrystals, such as metal organic frameworks (MOFs) ${ }^{72}$ and titania monolayers $\left(\mathrm{Ti}_{0.87} \mathrm{O}_{2}\right)^{73}$, into the charge- 
a

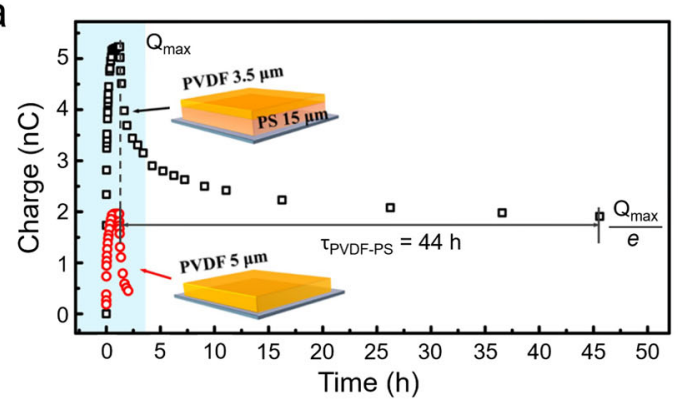

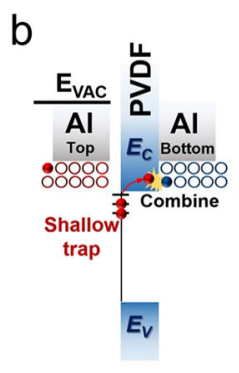

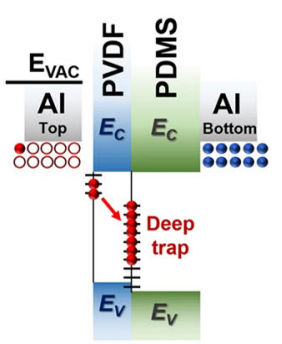

C
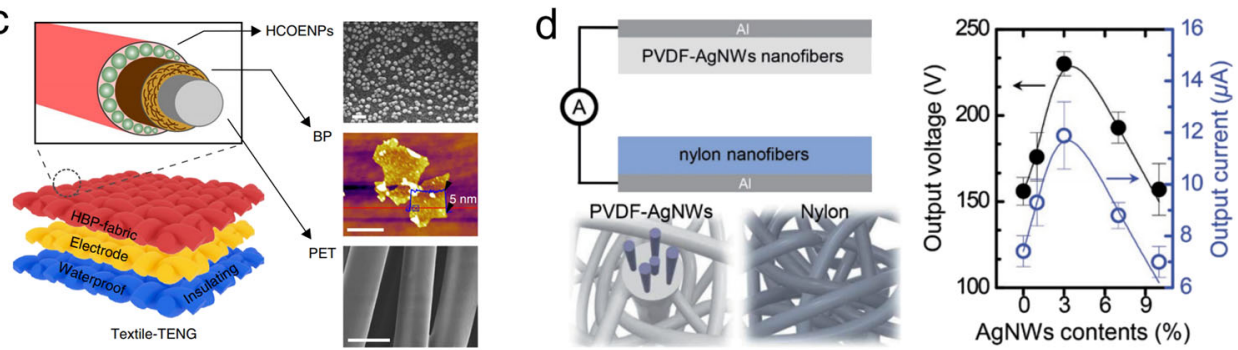

Fig. 3 Utilization of the charge-trapping layer for power enhancement. a Accumulation and decay processes of the trapped triboelectric charges with/without the PS dielectric interlayer (modified from ref. ${ }^{63}$, copyright permission from the American Chemical Society). b Chargetrapping mechanism with a PDMS deep-trap interlayer (modified from ref. ${ }^{65}$, copyright permission from Elsevier). c Black phosphorus layer coated with hydrophobic nanoparticles used for trapping the electrons (modified from ref. ${ }^{70}$, copyright permission from Nature Publishing Group). $\mathbf{d}$ Enhancement of charge trapping and charge induction by adding silver nanowires (Ag NWs) into the PVDF nanofiber matrix (modified from ref. ${ }^{71}$, copyright permission from Wiley-V(H).

generating layers enhanced the TENG output power due to the synergetic effects between efficient electron capture and the increase in the dielectric constant of the nanocrystals.

\section{Charge-collecting layer}

When two dielectric surfaces come into contact, the generated charges are electrostatically driven to flow from one electrode to another electrode ${ }^{28}$. When an electrode contacts a dielectric, the electrode acts as both a chargegenerating layer and a charge-collecting layer ${ }^{42}$. Solid and flexible electrodes, including metals ${ }^{74,75}$, graphene $^{18}$, and indium tin oxide (ITO), are well established ${ }^{17,76}$. Relatively, the practical application of deformable electrodes is still very limited because deformable TENGs and TENG sensors require reliable deformability in electrodes. Some of the approaches to produce deformable electrodes and their uses for stretchable TENGs are introduced in this section.

There have been extensive studies on deformable electrodes. In-plane serpentine metal electrodes and out-ofplane buckled electrodes have been widely used in stretchable electronic devices ${ }^{77-81}$. Considering the large dimensions of TENGs and the high mechanical impacts/ frictions applied on the device, such wavy metal electrodes are not appropriate for general use in TENGs. Conductive elastic nanocomposites are alternative electrodes because low-cost printing over a large area is possible, and the composite is mechanically stable under repeated mechanical impacts and friction ${ }^{82-84}$. Elastomer composites made of metal flakes or metal nanosheets have often been used to fabricate deformable TENGs ${ }^{85,86}$. The Au nanosheet elastomer composite electrode has stable electrical properties under various mechanical environments $^{84}$. The Au nanosheets were embedded in a PDMS matrix and used as a charge-collecting layer in TENGs $^{65,87}$ (Fig. 4a). During the 10,000 repetitive cycles of elongational strain (30\%), the initial voltage output was held constant (Fig. 4b).

Liquid metals are another choice for highly stretchable electrodes ${ }^{88-90}$. Due to their fluidic nature at room temperature, liquid metals can be injected into a microchannel fabricated in an elastomer matrix. Figure $4 \mathrm{c}$ shows a single-electrode $\mathrm{TENG}^{91}$. When human skin separates from a rubber surface, charges are generated, and electrons flow from the liquid metal to the ground. When human skin approaches the rubber, the electrons flow in the reverse direction to the liquid metal. The single-electrode TENG is highly stretchable and deformable without causing rupture of the electrode ${ }^{91}$ (Fig. 4d). Not only the liquid metal but also the liquid-state conducting polymer, a solution of poly(3,4-ethylenedioxythiophene):poly(styrenesulfonate) (PEDOT:PSS) and physiological saline, was reported as a shape-adaptive and stretchable charge collector in TENGs ${ }^{92,93}$. 


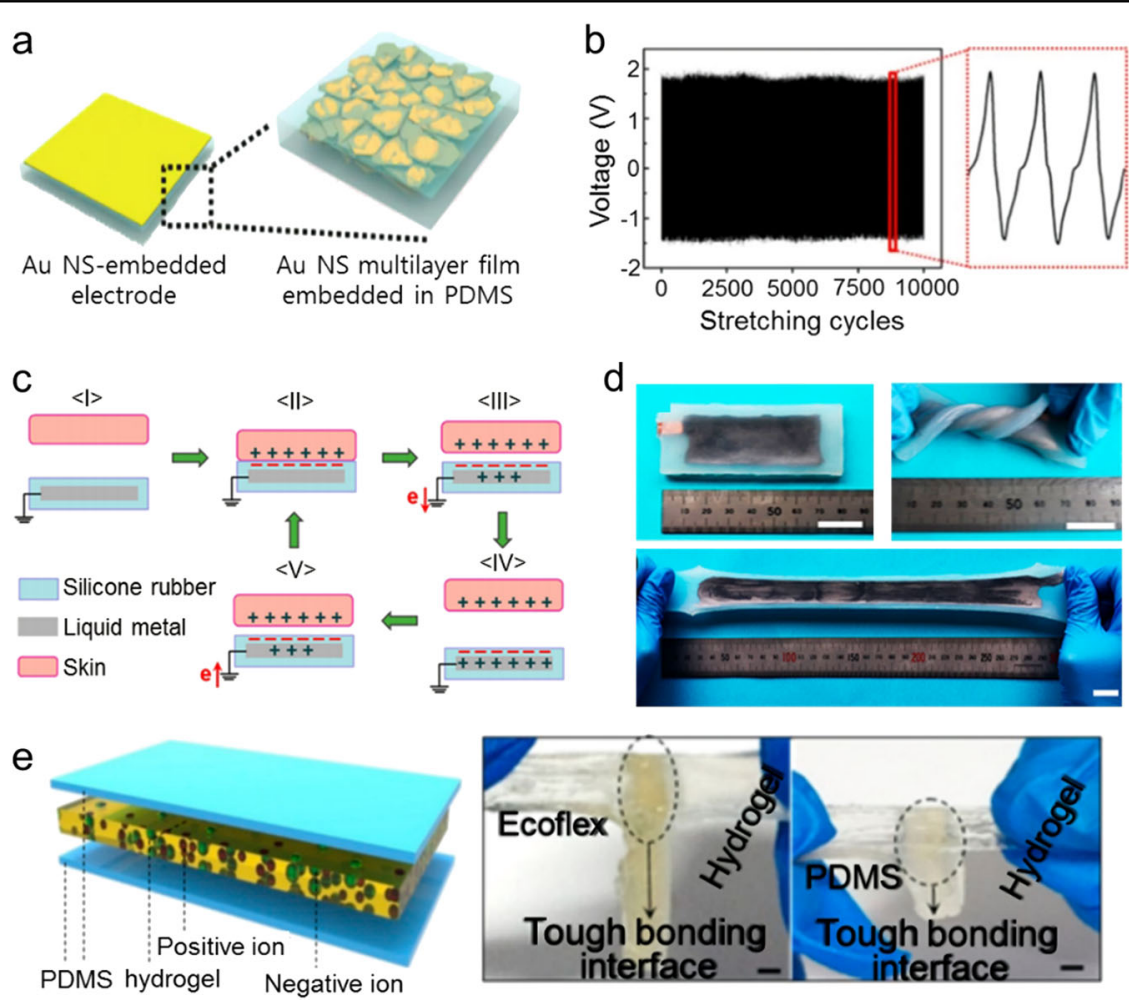

Fig. 4 Utilization of stretchable materials for charge collection. a Stretchable electrode made by embedding Au nanosheets in a PDMS matrix and $\mathbf{b}$ stable voltage output when cyclic elongational strain was applied (modified from ref. ${ }^{87}$, copyright permission from Elsevier). $\mathbf{c}$ Working mechanism of a TENG device by using a liquid metal electrode and $\mathbf{d}$ the high stretchability of the device (modified from ref. ${ }^{91}$, copyright permission from American Chemical Society). e lonic conductor consisting of PAAm-alginate hydrogel and PDMS. The interface was modified by benzophenone for strong bonding between the layers (modified from ref. ${ }^{96}$, copyright permission from the American Chemical Society).

Electrolyte gels such as hydrogels and ion gels allow both high stretchability and high transparency. Hydrogels are composed of a hydrophilic polymer network swollen with water or ionic aqueous solution ${ }^{94,95}$. The weak adhesion between the conductive electrolyte and the electrification elastomer layer can be an issue in TENGs in terms of mechanical reliability. Liu et al. demonstrated a strong interface between the polyacrylamide-sodium alginate (PAAm-alginate) hydrogel and the silicone rubber ${ }^{96}$. The hydrogel was sandwiched between two elastomer films containing benzophenone. Benzophenone formed covalent bonds between the hydrogel and the silicone rubber (Ecoflex); hence, the layers maintained a strong interface under a large elongation (Fig. 4e). The output voltage of the single-electrode TENG showed no change during 5,000 cycles of repeated contact-separation motions. If the effect of humidity on electrolyte-based TENGs can be solved, the electrolyte will be a promising stretchable and transparent electrode for TENGs ${ }^{97,98}$.

\section{Charge-storage layer}

To use the generated triboelectricity as a power supply, the power must be stored in an energy-storage unit, and the AC power from TENGs must be converted to DC power. Typically, a circuit board containing a capacitor for power storage and a bridge rectifier for DC conversion has been used ${ }^{99}$. Since the absolute power of TENGs is small, application of these materials has been concentrated on wearable mobile sensors that do not require high electric power. Flexible or stretchable capacitors and rectifiers are suitable for mechanically compliant wearable devices. In addition, they should be integrated all-in-one on a flexible or stretchable substrate. This aspect of TENGs has rarely been investigated. This section introduces studies on the fabrication of flexible stretchable capacitors and rectifiers that require new types of materials.

A straightforward way to store triboelectric energy is to incorporate a rechargeable battery with a TENG. Nan et al. reported a high energy conversion efficiency from a TENG to a lithium-ion battery (LIB) ${ }^{100}$. The schematic in Fig. 5a represents a power-storage system consisting of a TENG and an LIB. Various cathode materials were reported, including $\mathrm{Li}_{3} \mathrm{~V}_{2}\left(\mathrm{PO}_{4}\right)_{3}{ }^{100}, \mathrm{LiFePO}_{4}(\mathrm{LFP})^{101,102}$, $\mathrm{LiMn}_{2} \mathrm{O}_{4}(\mathrm{LMO})^{103}$, and $\mathrm{LiCoO}_{2}(\mathrm{LCO})^{104}$, to charge the triboelectric energy. They obtained a high conversion 

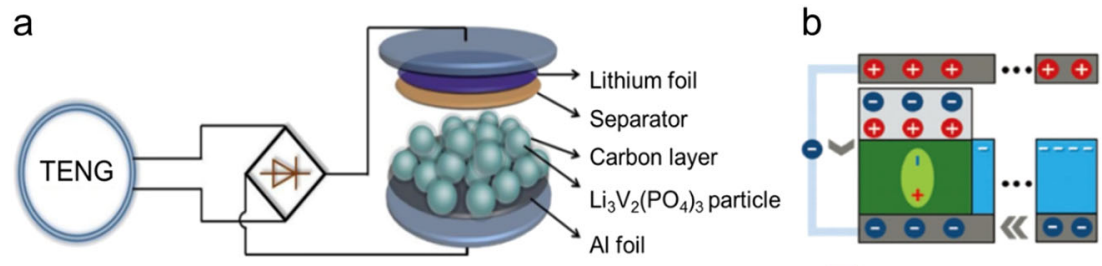

C

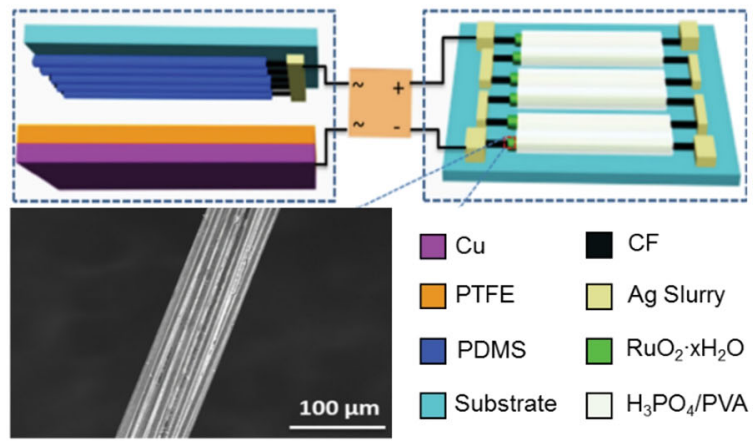

b

Dielectric material

Triboelectric material

d

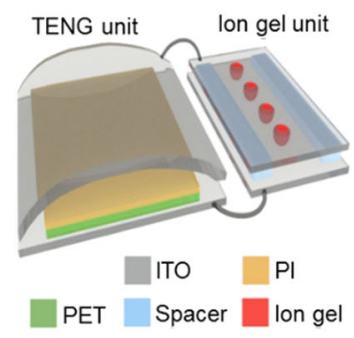

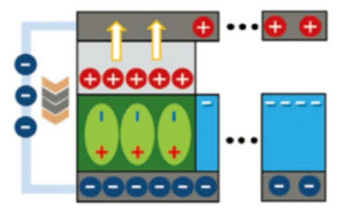

TENG electrode

Capacitor electrode

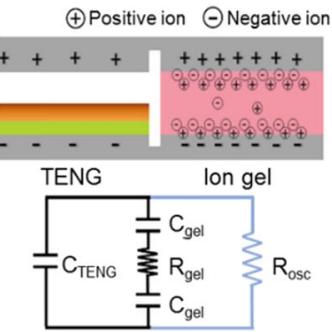

Fig. 5 Capacitive materials for charge storage in TENG. a Integration of TENGs and lithium-ion batteries (LIBs) based on a $L i_{3} V_{2}\left(\mathrm{PO}_{4}\right)_{3} / \mathrm{C}$ nanocomposite (modified from ref. ${ }^{100}$, copyright permission from the American Chemical Society). b Dielectric capacitor with a P(VDF-TrFE) used for storing and releasing the electrostatic charges (modified from ref. ${ }^{110}$, copyright permission from Wiley-VCH). c Flexible and weavable self-charging power system by combining a fiber-based supercapacitor with TENGs (modified from ref. ${ }^{115}$, copyright permission from Wiley-VCH). $\mathbf{d}$ Triboelectric voltage modulation by printed ion gel patterns (modified from ref. ${ }^{118}$, copyright permission from Elsevier).

efficiency (more than 80\%). However, charging was performed with a rotary TENG at a high frequency $(1300 \mathrm{~Hz})$ of rotary sliding ${ }^{105,106}$. A conventional Li-ion battery requires a current at the $\mathrm{mA}$ level for charge/discharge ${ }^{106}$; hence, direct charging in an LIB requires a coil transformer that increases the amplitude of the AC output current or a high-frequency stimulation (such as in highspeed rotating drum). Unfortunately, direct charging of a battery with a TENG is not practical due to the low current density that cannot overcome the charging energy barrier.

Thus far, the energy from TENGs has been stored in capacitors. A capacitor can be fabricated simply by inserting a dielectric layer between two electrodes. Since the dielectric capacitance is expressed by the equation $C=k \varepsilon_{o} A / d$, where $k, \varepsilon_{o}, A$, and $d$ are the dielectric constant, vacuum permittivity, electrode area, and dielectric thickness, respectively, the performance is easy to characterize by the dimensions and inherent permittivity. The fast charge/discharge performance of inorganic dielectric materials can be used for TENG capacitors to achieve a high-power density. Due to the poor flexibility of inorganic dielectric film capacitors, the growth of dielectric materials on flexible organic substrates and metal foils has been investigated ${ }^{107,108}$. Liang et al. have grown an ITO film on fluorophlogopite (FMica) to use as a high-temperature-resistant, bendable substrate to grow a barium zinc titanate (BZT) dielectric layer on ITO $^{109}$. However, brittle ceramic capacitors still have limited use in flexible, stretchable devices. High- $k$ polymer dielectric films are good candidates for flexible capacitors. Chung et al. reported a capacitor-integrated TENG in which the capacitor made of PVDF-TrFE as the dielectric layer stored electrostatic charges and released the charges due to the Leyden jar effect ${ }^{110}$. As shown in Fig. 5b, the dielectric layer (green) between a TENG electrode (gray) and a capacitor electrode (light gray) was charged through the triboelectric layer (blue) and induction onto the TENG electrode. When the charged metal contacted the TENG electrode, the metal-to-metal contact released the stored charges and generated a large current $(4.3 \mathrm{~mA})$.

The electrolyte is an excellent capacitive material due to the formation of an electric double layer (EDL). Depending on the ion concentration, the capacitance can be controlled from $\mathrm{nF}$ to hundreds of $\mu \mathrm{F}^{111}$. The disadvantages of electrolyte capacitors are the frequency dependence and the temporal relaxation of the capacitance. Since the contact frequency of TENGs is typically below $100 \mathrm{~Hz}$, the frequency dependence of the electrolyte (constant up to $\sim 100 \mathrm{~Hz}$ ) is not a problem in TENGs $^{112}$. The advantages of using an electrolyte capacitor are printability and patternability, as well as high flexibility and deformability. Combining TENGs and electrolyte capacitors can lead to new power sources, such as TENG-powered supercapacitors ${ }^{113-115}$. Wang et al. fabricated a flexible and wearable self-charging power system consisting of a fiber-based supercapacitor and a 
fiber-based TENG (Fig. 5c) ${ }^{115}$. Carbon fibers covered with $\mathrm{RuO}_{2} \cdot \mathrm{xH}_{2} \mathrm{O}$ were assembled in a bundle, as shown in Fig. 5c. $\mathrm{RuO}_{2} \cdot \mathrm{xH}_{2} \mathrm{O}$ is a widely used material for electrochemical capacitors due to facile transport pathways for both protons and electrons, highly reversible redox reactions, wide electrochemical windows, and a long cycle life ${ }^{116}$. The specific capacitance of $\mathrm{RuO}_{2} \cdot \mathrm{xH}_{2} \mathrm{O}$ was much higher $(1000 \mathrm{~F} / \mathrm{g})$ than the specific capacitance of bare ruthenium oxide $(720 \mathrm{~F} / \mathrm{g})^{117}$. The fiber supercapacitor was charged by contact separation of the PDMS and PTFE layers. This fiber-type supercapacitor is a possible route to flexible self-charging power systems.

An additional advantage of using an electrolyte capacitor is the precise modulation of the output voltage by adjusting the capacitance of the electrolyte ${ }^{118}$. Lee et al. printed ion gel patterns on an electrode and used them as capacitors to modulate the maximum output voltage. An ion gel consisting of an ionic liquid (EMIM-TFSI) and a polymer (PVDF-HFP) was sandwiched between ITO electrodes that were connected to the TENG electrodes (Fig. 5d). During contact and separation in TENGs, the charges on the electrodes developed EDLs in the ion gel. The circuit diagram summarizes the parallel connection of the TENG capacitance $\left(\mathrm{C}_{\mathrm{TENG}}\right)$, the ion gel capacitance $\left(\mathrm{C}_{\mathrm{gel}}\right)$, and the oscilloscope resistance $\left(\mathrm{R}_{\mathrm{OSC}}\right)$. The relaxation time $(\tau=R C)$ of the ion gel determined the measured voltage profile. Lee et al. changed the spike-like voltage peaks in TENGs to square voltage profiles ${ }^{118}$. The addition of a $\mathrm{Ru}(\mathrm{bpy})_{3}\left(\mathrm{PF}_{6}\right)_{2}$ luminophore in the ion gel led to electrochemical luminescence. The voltage profile became more sluggish due to the increased capacitance by the redox reaction of $\mathrm{Ru}^{2+}$. Since the ion gel could be readily printed and patterned by UV, the capacitance of a single pattern was tunable according to the size of the pattern. In addition, the maximum voltage and the overall voltage profile were controlled separately through the serial connection and parallel connection of the ECL gel dot patterns.

Self-powered mobile healthcare sensors may be a major application of TENGs. These sensors require stretchable dielectric layers both for the TENG unit and for the capacitor. Kong et al. quantified the capacitance of commercially available elastomeric dielectrics such as PDMS, hydrogenated polystyrene-block-poly(ethylene butylene)block-polystyrene (H-SEBS), elastic PVDF-HFP, and polyurethane $(\mathrm{PU})^{119}$. PDMS and H-SEBS exhibited dielectric behavior similar to that of conventional polymer dielectrics, whereas PVDF-HFP and PU showed high capacitances because of the mobile ions present in the elastomers. In addition, the capacitance was stable in a broad temperature range $\left(20-160^{\circ} \mathrm{C}\right)^{119}$. These elastomeric dielectrics can be useful for application in stretchable TENGs and TENG sensors. The achievement of a high- $k$ stretchable dielectric has rarely been studied.
Nanocomposites with high- $\mathrm{k}$ inorganic nanomaterials and stretchable electrolytes are two possible approaches. Both approaches need further studies for practical uses.

\section{Material approaches for TENG sensors}

The electrical signals obtained in TENGs can be used not only for electric power generation but also for sensing momentary stimuli. Research on the quantitative recognition of mechanical stimuli (touch, pressure, stretching, and torsion) has mainly been carried out with TENGs; in recent years, applications for chemical sensing have also been attempted. In this section, we describe the design of materials for TENG sensors and methods to deconvolute the signals in multimodal sensors.

\section{Material design for mechanical stimuli sensors}

Sensors that can detect mechanical stimuli are critical components in electronic skin, touch screen, healthcare devices, and habit-recognition security systems ${ }^{120}$. Mapping and imaging of stimuli are important for smart displays, flexible and wearable electronics, and human-robot interfaces ${ }^{121}$. Since the TENG sensors generate potential signals, sensing does not require external electric power, resembling the action potential generated in human mechanoreceptors. This potential generation can be effective in power savings when the entire sensing system needs a large number of sensors, as in the human tactile sensory system containing a tremendous number of mechanoreceptors ${ }^{122}$. The first material requirement for the sensor is to acquire a quantitative relationship between the sensing signals and the stimulation. The second material requirement is to obtain long-term reliability of the sensing signals.

Quantitative conversion of the potential signals into external tactile stimuli (pressure and contact) has been mostly sought by implementing the microstructures on the contact surfaces to obtain large output signals at low pressures $^{74,123-127}$. Zhu et al. proposed a flexible sensitive tactile sensor that can detect a wide range of contact pressures from 0.03 to $10 \mathrm{kPa}$. They used vertically aligned FEP nanowires on contact surfaces ${ }^{123}$. This sensor was integrated with signal-processing circuits to detect the hand touch on the door handle and turn on a motionactivated lighting system. Differentiation between static pressures and dynamic pressures is important to recognize human body movements in daily activities. Lin et al. successfully recognized both static and dynamic pressures with a single TENG sensor ${ }^{124}$ (Fig. 6a). Due to the microstructure on the PDMS contact surface, the sensor showed a low detection limit (2.1 Pa), high sensitivity $\left(0.31 \mathrm{kPa}^{-1}\right)$, and fast response $(<5 \mathrm{~ms})$. The stable elastomer materials provided excellent mechanical stability (30,000 cycles). By integrating multiple sensor units on the contact surface, the local pressure distribution was 

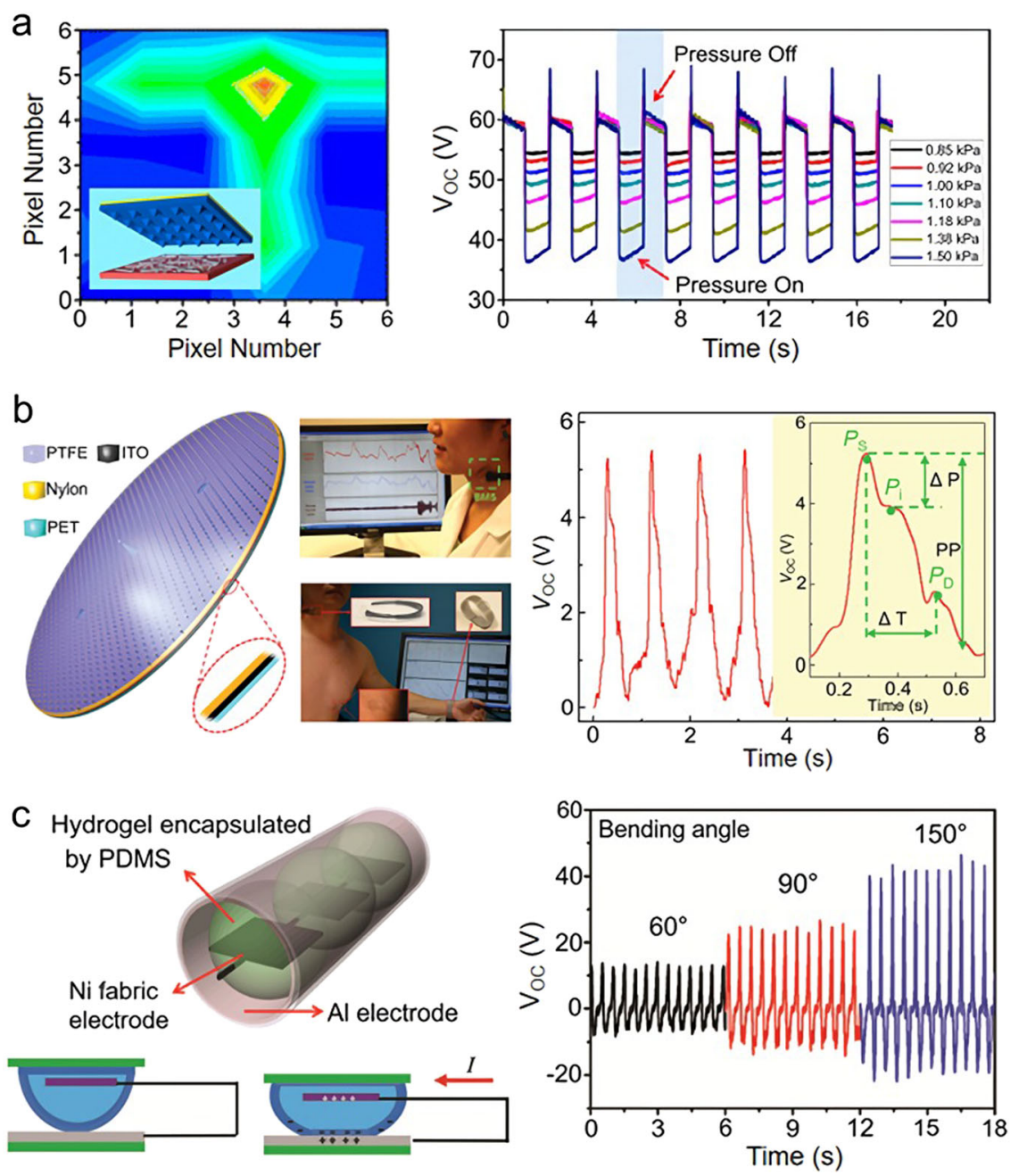

Fig. 6 Mechanical stimulus of TENG sensors. a Pressure mapping with a self-powered triboelectric pressure sensor array and the changes of the open-circuit voltage under variable pressures (modified from ref. ${ }^{124}$, copyright permission from the American Chemical Society). $\mathbf{b}$ Eardrum-inspired triboelectric sensor detecting both cardiovascular arterial pulse and throat sound vibrations (modified from ref. ${ }^{127}$, copyright permission from WileyVCH). c Hydrogel-based triboelectric sensors for the detection of bending, stretching, and twisting motions (modified from ref. ${ }^{133}$, copyright permission from Wiley-V(H).

mapped. Ren et al. proposed a TENG-based tactile sensor that can detect both normal and tangential forces ${ }^{125}$. The elastic tiny burr arrays on the contact interfaces not only can detect both normal and tangential forces but also can distinguish the direction of applied tangential forces with high sensitivity. Because the triboelectric phenomenon is the contact electrification, the TENG-based sensors are highly sensitive to low pressure and thus are suitable for detecting minute movements or biological pulses in the human body ${ }^{74,126,127}$. Pu et al. developed a TENG-based motion sensor with FEP nanowires on its contact surface. The sensor could detect the mechanical motions of the skin around the corners of the eyes so that it could recognize different eye motions ${ }^{126}$. Yang et al. fabricated a contact surface with poly(tetrafluoroethylene) (PTFE) nanowires, inspired by the structure of the human eardrums (Fig. 6b). They reported a bionic membrane sensor that not only monitored low-frequency cardiovascular arterial pulses but also acquired high-frequency throat sounds and vibrations ${ }^{127}$.

In addition to simple pressure stimulation, TENG sensors can detect other dynamic mechanical stimuli, such as shear, stretch, bend, and twist. These sensors are designed to be compliant with the deformation of an object and not to interfere with the stimulation. The main use of these stretchable TENG-based sensors is monitoring human body motions in joints, limbs, and muscles ${ }^{128-130}$. Jin et al. designed a finger-motion sensor to detect bending and unbending of the finger joints in real time ${ }^{128}$. The sensor was composed of FEP and PET layers. Similarly, Lu et al. 
designed a stretchable strain sensor with a ladder structure of the PTFE film and a stretchable aluminum electrode fabricated on an elastic rubber substrate ${ }^{129}$, and Chen et al. proposed an on-skin stretchable TENG sensor consisting of an elastic dielectric film and a carbon grease electrode that can perform tactile sensing at high strain without being disturbed by human movements ${ }^{130}$. These sensors were attached to the human skin and used to monitor the movement of joints and muscles. To attain high conductivity and prevent a decrease in the conductivity of the sensor electrode in the stretched state, ion gels have been proposed as an electrode for the TENGbased sensors ${ }^{131-133}$. Pu et al. used a polyacrylamide (PAAm-LiCl) hydrogel containing lithium chloride as an electrode and demonstrated a skin-like tactile sensor ${ }^{131}$. This soft sensor was transparent and highly stretchable (stretchable up to $1160 \%$ ) and could sense pressures as low as $1.3 \mathrm{kPa}$. Hydrophobic ion gels have also been utilized in TENG sensors. Zhao et al. used the poly(2-acrylamido-2-methyl-1-propanesulfonic acid) (PAMPS)-based ion gel as both the electrode and triboelectric contact layer to fabricate a transparent stretchable strain sensor $^{132}$. They inserted a micropatterned PDMS layer between the PAMPS ion gel electrode and the contact layer. The contact area between the PDMS micropattern and the ion gel layer changed according to the applied strain; thus, the magnitude of the electrical signals had a quantitative correlation with strain. Xu et al. fabricated a cylindrical stretchable $\mathrm{Al}$ electrode containing polyvinyl alcohol (PVA) hydrogel balls encapsulated by PDMS ${ }^{133}$ (Fig. 6c). A Ni fabric electrode was placed inside the hydrogel ball and wired to the $\mathrm{Al}$ electrode. The changes in the contact area between the PDMS and the Al electrode resulted in corresponding changes in the magnitude of the potential; thus, the sensor could provide quantitative information on body motions (bending, twisting, and stretching).

\section{Material design for signal selectivity}

Mechanical stimulation often simultaneously includes several force components, such as normal force, pure shear, and torque. The sensors for electronic skin and haptic devices typically hold information about pressure and $\operatorname{strain}^{134-137}$. For practical use of the sensor, the sensor should selectively respond to a specific force component or deconvolute specific signals from a bundle of mixed signals. For instance, the finger-motion strain sensor must not respond to touch; otherwise, the sensor will give incorrect operation signals. Signal selectivity is crucial in biological sensors for healthcare monitoring. The issue in the haptic device is the real-time recognition of the motion dynamics. The sensor should transfer the speed information of a mechanical stimulation for motion control of an object. However, in some other cases, the sensor should be selectively dependent on the total strain or total pressure regardless of the motion dynamics. Therefore, the dynamic information and the strain/pressure information should be obtained separately.

Although the signal selectivity is critical for practical uses, it has rarely been investigated in TENG-based sensors. One way to obtain multiple information selectively is by combining different sensors. For example, a combination of a TENG sensor and an EMG (electromagnetic generator) sensor enabled detection of the magnitude and the velocity of a body motion ${ }^{138}$. The peak voltage value in the TENG sensor provided information on the maximum force, while the peak current in the EMG sensor provided the maximum velocity. To make the sensor system simple, material design is necessary for proper signal deconvolution from a multimodal sensor. $\mathrm{Li}$ et al. demonstrated a dual-mode sensor that can separate touch from pressure and provide hardness information ${ }^{139}$ (Fig. $7 \mathrm{a})$. The sensor could determine the hardness of a contact material by analyzing the shape of the current profile obtained at the moment of contact. Hard materials created sharp rectangular current peaks, whereas soft materials generated broad current peaks. Since the open-circuit voltage changed linearly with the external force (Fig. 7b), simultaneous detection of pressure and hardness was realized when a foreign object was pressed on a TENG sensor. Very recently, Choi et al. reported a stretchable TENG sensor that could extract information about pressure, strain, moving distance, and moving velocity of the sensor ${ }^{140}$. They fabricated a multilayer stretchable sensor that could separate signals from the sliding mode and the contact mode (Fig. $7 \mathrm{c})$. The key to the success was blocking the signal interference when stretching and pressing were exerted simultaneously. The electrode for the sliding mode was placed in the inner part, and the electrode of the contact mode was placed at the upper part. For the sliding mode, a PTFE thin film was used due to its low friction coefficient. To block the signal interference, they placed a stretchable electrode beneath the top PDMS surface. The stretchable electrode was made of a PDMS/Au nanosheet composite film. Figure $7 \mathrm{~d}$ shows the potential peaks measured from the sliding mode (measurement 1 ) and from the contact mode (measurement 2). The sliding mode responded only to stretching without any signal change by pressing events, whereas the contact mode showed large peaks by pressing but negligible peaks by stretching.

\section{Material design for chemical sensors}

Contact electrification can change when chemicals are adsorbed on the contact surface. The electrical outputs should be dependent on the amount and the chemical species of the adsorbent. In situ composition changes through doping may be another possible way to cause changes in the triboelectric outputs. These characteristics 

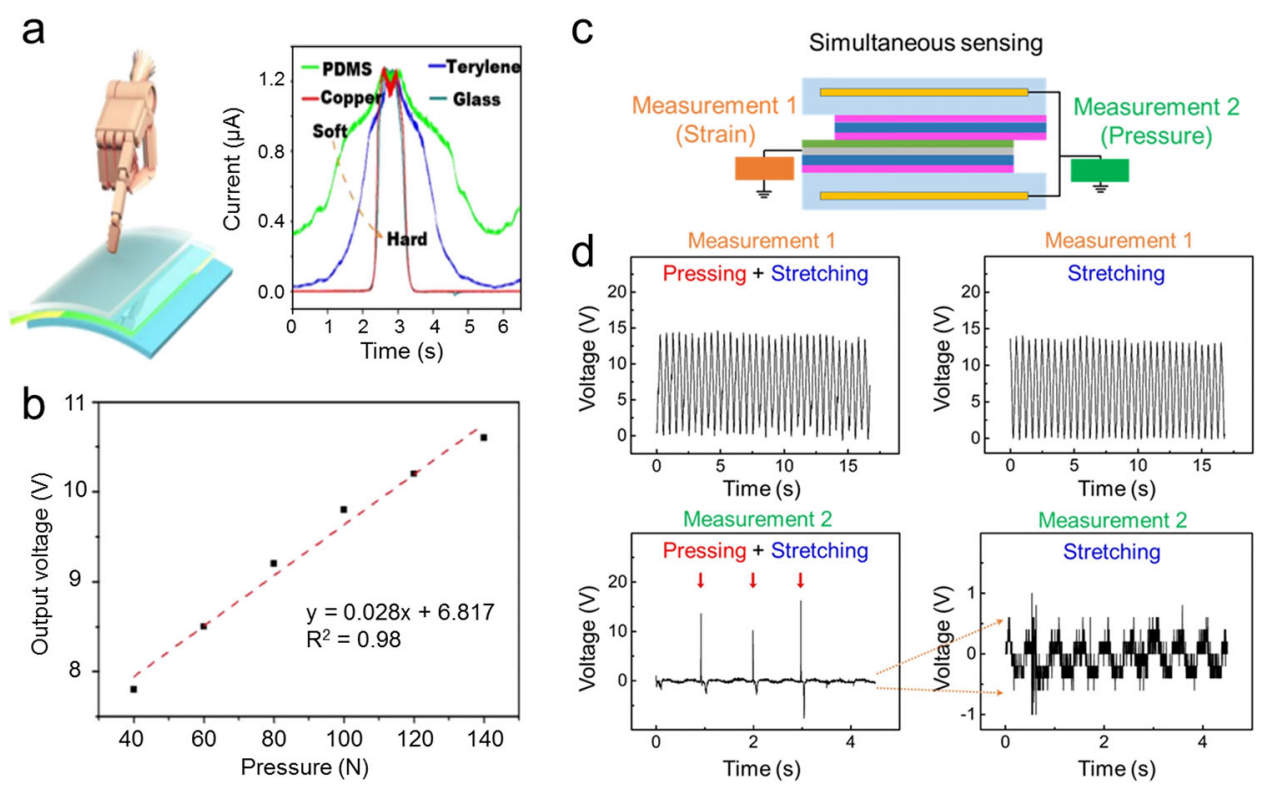

Fig. 7 Multimodal TENG sensors. a Dual-mode TENG sensor detecting touch and hardness of the material by analyzing the shape of the current profile and $\mathbf{b}$ the linear relationship between the sensor output voltage and the external pressure (modified from ref. ${ }^{139}$, copyright permission from the American Chemical Society). c Stretchable TENG sensor discriminating pressing and elongation in one system and $\mathbf{d}$ its voltage output responses to pressure (Measurement 1) and strain (Measurement 2) (modified from ref. ${ }^{140}$, copyright permission from Elsevier).

introduce the possibility of fabricating TENG-based chemical sensors, either in a liquid phase or in a gas phase.

To endow the chemical selectivity of the sensor, the contact surface should be modified to capture a target chemical selectively. Lin et al. demonstrated a TENGbased $\mathrm{Hg}^{2+}$ ion sensor ${ }^{141}$. They coated Au nanoparticles on the contact surface. 3-Mercaptopropionic acid (3MPA), which is known to selectively bind to $\mathrm{Hg}^{2+}$ ions, was self-assembled on the Au nanoparticles. They found that the sensor responded selectively to the concentration of $\mathrm{Hg}^{2+}$ ions when it was dipped in a solution containing various metal ions. When the triboelectric output was measured after drying the sensor in air, the triboelectric output from the sensors decreased as the concentration of $\mathrm{Hg}^{2+}$ increased. A similar approach was used to detect organic molecules. Titanium dioxide $\left(\mathrm{TiO}_{2}\right)$ nanowires were coated on a Ti electrode, and their surfaces were modified with $\beta$-cyclodextrin $(\beta-C D)$, which has a specific binding to phenol ${ }^{142}$ (Fig. 8a). The output signal decreased with increasing concentrations of phenol, with a sensitivity of $0.01 \mu \mathrm{M}^{-1}$ and a sensing range of $10-100 \mu \mathrm{M}$. By placing the sensor in phenol-containing wastewater, the sensor not only detected the phenol concentration but also electrochemically purified the wastewater into clean water. Liquid-solid contact electrification, instead of conventional solid-solid electrification, opens a new possibility in chemical sensing. Choi et al. proposed a concept of a smart pipette tip to measure the electrolyte concentration of the dispensed solution.
They utilized the spontaneously generated electric signals during the pipetting process ${ }^{143}$. The smart pipette tip was used for in situ prediction of both the concentration and morphology of palladium (Pd) nanoparticles in the electrolyte. Likewise, a microfluidic ion concentration sensor was demonstrated by Jeon et al. ${ }^{144}$. The electrical signals generated through the contact between a polymeric channel surface and a moving fluid were used to detect the ion concentration in the fluid (Fig. 8b). The output signal decreased quantitatively with increasing concentration of sodium chloride $(\mathrm{NaCl})$ in water with a good concentration resolution and reliability in the range from $5 \times 10^{-3}$ to $0.1 \mathrm{M}$.

TENG-based gas sensors use the changes in output signal when chemicals are adsorbed on the contact surface. Cui et al. reported an ammonia $\left(\mathrm{NH}_{3}\right)$ gas sensor by using the absorption and desorption in polyaniline (PANI) nanofibers ${ }^{145}$ (Fig. 8c). PANI has been known as a highly sensitive $\mathrm{NH}_{3}$ gas-sensing material ${ }^{146}$. When PANI is exposed to $\mathrm{NH}_{3}$ gas, it is deprotonated, and its conductivity decreases. In the TENG sensor, the PANI nanofibers worked both as $\mathrm{NH}_{3}$ gas-sensing materials and as triboelectric electrodes. The sensor had a good resolution with a detection limit of $500 \mathrm{ppm}$ and was insensitive to other volatile gases. Similarly, a hydrogen $\left(\mathrm{H}_{2}\right)$ gas sensor was fabricated ${ }^{147}$. Zinc oxide nanorods decorated with $\mathrm{Pd}$ nanoparticles were used as both the $\mathrm{H}_{2}$ sensing material and the contact material in the sensor. The chemisorbed oxygen ions $\left(\mathrm{O}^{2-}\right)$ formed by the reaction 
a

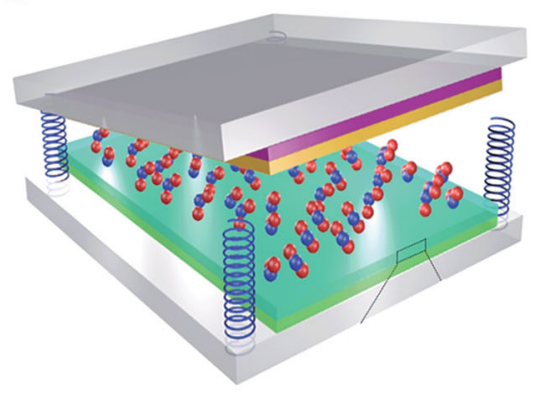

C

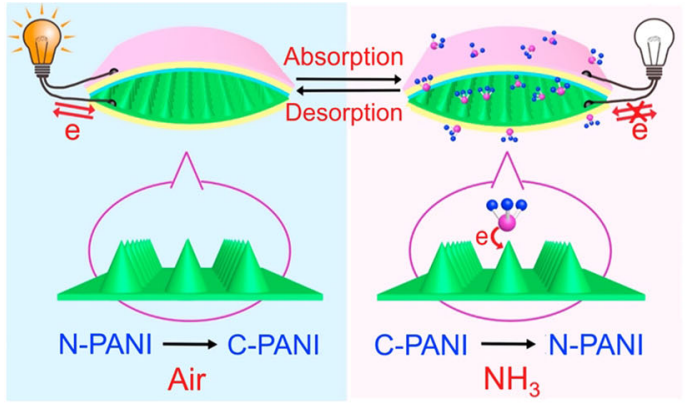

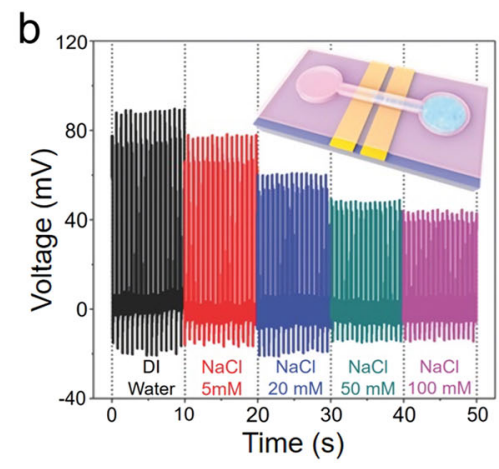

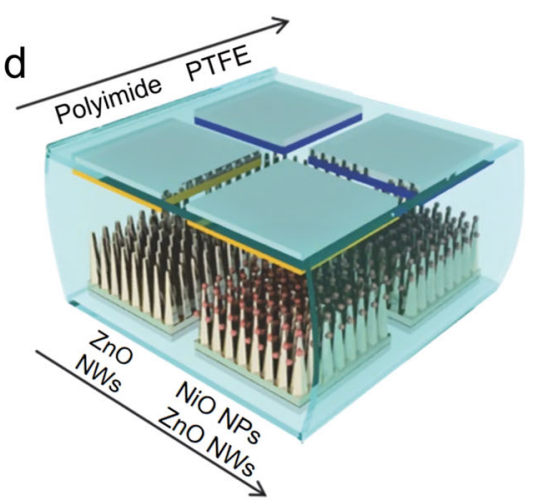

Fig. 8 TENG-based chemical sensors. a Phenol detection in wastewater by using triboelectrification with a $\beta$-cyclodextrin surface (modified from ref. ${ }^{142}$, copyright permission from the Royal Society of Chemistry). $\mathbf{b}$ lon concentration sensor by using the liquid-solid contact electrification in a microfluidic system (modified from ref. ${ }^{144}$, copyright permission from Wiley-VCH). c Mechanism of sensing $\mathrm{NH}_{3}$ gas in a PANI-based TENG gas sensor (modified from ref. ${ }^{145}$, copyright permission from Elsevier). d Self-powered electronic nose based on the triboelectrification and the heterogeneous catalytic reaction (modified from ref. ${ }^{148}$, copyright permission from Wiley-VCH).

between the $\mathrm{Pd} / \mathrm{ZnO}$ electrode and the oxygen molecules in the air reacted with $\mathrm{H}_{2}$ molecules and formed water $\left(\mathrm{H}_{2} \mathrm{O}\right)$ molecules. The higher $\mathrm{H}_{2}$ concentration in the air resulted in more $\mathrm{H}_{2} \mathrm{O}$ molecules formed on the contact surfaces, causing a decrease in the electrical output of the sensor. In this mechanism, the sensor showed a minimum detecting concentration of $10 \mathrm{ppm}$ and a short response time of $100 \mathrm{~s}$.

Gas sensors with a high sensitivity and selectivity similar to those of the human nose, called "electronic noses", have been studied and are expected to provide sustainable healthcare services. Based on TENGs, electronic noses that are capable of detecting several gases without the need for external power have been reported. Kim et al. reported an electronic nose with a highly selective gas detection capability ${ }^{148}$ (Fig. 8d). They fabricated arrays of $\mathrm{ZnO}$ nanowires that were coated with nickel oxide $(\mathrm{NiO})$ nanoparticles and used them as the contact materials. The catalytic oxidation between $\mathrm{NiO}$ nanoparticles and gas molecules generated electrons. Electron transfer to the semiconducting $\mathrm{ZnO}$ nanowires increased the triboelectric charge density at the contact surface, thereby increasing the output signal with increasing gas concentration. Since the catalytic reactions occurring on the
$\mathrm{ZnO}$ nanowires and $\mathrm{NiO}$ nanoparticles differ depending on the type of gas molecules, these electronic noses distinguished between four volatile organic compound (VOC) gases with a detection limit of $0.1 \%$. A flexible electronic nose that can detect ethanol in the air was realized from the PANI/PTFE/PANI sandwich nanostructure $^{149}$. When the PANI was exposed to ethanol, the interaction between nitrogen atoms of the PANI and the hydroxyl $(-\mathrm{OH})$ groups of ethanol altered the conformation of PANI chains and affected charge transportation through the chain. The degree of rearrangement in the PANI chain depended on the concentration of ethanol, thus changing the triboelectric output. Due to its high sensitivity to ethanol, this electronic nose could be used as a mobile alcohol sensor without requesting external electrical power.

\section{Challenges in materials of TENGs}

Although the triboelectric effect is one of the most frequently experienced in everyday life, research on energy harvesting and sensor applications has been conducted in recent years since the first report of TENGs in 2012. The performance of TENG devices and TENG sensors has made tremendous advances during the short 
period of years since their first report ${ }^{150}$. TENG devices are expected to be a promising energy harvester for small devices, with efforts devoted to finding new mechanical power sources, combining with other types of devices, and exploring new applications. Advances in device performance are possible with a clear understanding of the charge generation mechanism; however, the mechanism of triboelectrification is still under debate ${ }^{151}$. It is generally believed that after two different materials are in contact, a chemical interaction is formed between the molecules on the surfaces, and the charges are transferred from one side to the other side to equalize the electrochemical potentials ${ }^{152}$. However, contact between two identical surfaces has also been reported to generate triboelectricity ${ }^{153}$. A possible explanation based on the thermionic-emission model has recently been reported, suggesting that the hotter material is positively charged and the cooler material is negatively charged through an electron transfer during contact electrification ${ }^{154}$. The question of "what are the transferred charges?" remains controversial for electrons ${ }^{155}$ and ions/molecules ${ }^{156}$.

Synthesis and discovery of new triboelectric materials $^{53,54,57}$ are necessary. In particular, positive triboelectric materials lack choices compared with the negative triboelectric materials ${ }^{51,157}$. Various lipids have been reported to be ranked as the highest positive materials in the triboelectric series, regardless of the type and position of the functional groups in molecules ${ }^{54}$. Wax molecules consisting of aliphatic carbons exhibit similar positive electrification. Considering that polyethylene, which has the same chemical bond structure, does not have strong positive surface charges, the surface electrification cannot be understood from the molecular structure, and the molecular orientation and crystal structure must be considered together. In addition to new material development, precise surface characterization of the existing triboelectric materials is also required in future studies. Although there have been many studies using PDMS, PVDF, PI, PET, and nylon as the contact surfaces, detailed information on the surface of the materials is not provided in articles. PDMS is a representative material used for negative charge generation; however, the surface molecules differ depending on the preparation process. It is well known that a thin liquid layer of unreacted small molecules is formed on the PDMS surface when cured at $80^{\circ} \mathrm{C}$ in the air. The unreacted chemicals disappear when cured at a high temperature $\left(180^{\circ} \mathrm{C}\right)$ in vacuum, and the modulus increases ten times ${ }^{158}$. The differences in the surface materials may cause a significant difference in the triboelectric property. The surfaces of the commercially available PI and PET films are often treated with other materials for easy film manufacturing or to prevent electrostatic charges. The lack of detailed information on the surface treatment results in the problem of general reproducibility in other groups even though the structure of the TENG device is simple.

Charge-trapping layers have not been studied in detail compared with charge-generating layers. A material that is superior in terms of both charge generation and charge trapping (such as $\mathrm{PI}^{64}$ or $\mathrm{PDMS}^{65}$ ) is more suitable for manufacturing a low-cost, high-performance TENG. More studies are needed on the fundamental principles of the charge trap mechanism in polymer films. Mechanically robust and stretchable electrodes can replace conventional brittle electrodes in TENGs. To overcome the humidity problems of the hydrogel electrodes, hydrophobic ion gels can be alternative stretchable transparent electrodes for TENG devices. In addition, the dynamics of the ionic molecules in the bulk and the electrode interface should be understood, which is in the beginning stage in theoretical studies. It is also important to improve the electrical stability of the wiring interface between the TENG electrodes and the other components (capacitors, rectifiers, and microprocessing units). Since metal wiring is not usable in connection with stretchable electrodes, stretchable interfacing conductive materials should be developed to guarantee high deformability of TENG devices without electrical disconnection under large strains $^{135}$.

TENG sensors are typically used as dynamic pressure sensors with spike-like signals ${ }^{19,159}$. Some studies demonstrated the possibility of static pressure sensors with a square-shaped constant voltage signal ${ }^{123,124}$. However, the square voltage profiles were obtained by using a high-impedance electrometer $(>1 \mathrm{G} \Omega)^{17,160}$, which is not realistic in typical electronic devices because the resistance of conventional devices is in the range of $\mathrm{k} \Omega$ to $10 \mathrm{M} \Omega$. Currently, without the aid of a highimpedance electrometer, TENG sensors cannot be used as static pressure sensors ${ }^{161}$. To detect both dynamic and static stimuli together, as in human sensory mechanoreceptors, combining a TENG sensor with a capacitive or resistive system is necessary ${ }^{162,163}$. However, TENGbased self-powered sensing can be useful in the human body, where dynamic movements and stimuli mainly occur. Implantable TENGs have been utilized to generate electricity from the biomechanical motions in the body ${ }^{15,164}$, or to acquire physiological information by obtaining electrical signals from the heart rate ${ }^{165}$, pulse $^{166}$, respiratory rate ${ }^{167}$, and bladder swelling ${ }^{168}$. As implantable TENGs and sensors are relatively new to wearable TENGs, biocompatible and desirable materials for implantable TENG devices must be developed. The hygroscopic environment, long-term stability, and stretchability of the device are additional material properties of implantable devices; thus, unconventional material studies are necessary to fabricate practical implantable TENG devices. In addition, in the case of 
tactile sensing, there is relatively little research on the use of the triboelectric phenomenon of the material itself ${ }^{169}$. Electrolytes can be used as a deformable capacitor that can finely tune the output voltage of TENGs. The spike-like AC high voltages in TENGs are not useful in electronics, and the voltage should be converted to a constant DC voltage. To tune the voltage and effectively harvest the charges in the electrolyte capacitor, an electrolyte material with a large charge relaxation time and charge resistance is needed $^{94,97}$. In TENG-based chemical sensors, sensing materials or systems that are not affected by environmental changes (temperature, humidity, ambient vibrations, etc.) are desirable. TENG-based gas sensors have utilized signal changes by incorporating gas molecules into triboelectric surfaces. The selective adsorption of gas molecules in polymers and metal oxides has been extensively stu$\operatorname{died}^{170,171}$. Adopting such materials as the substrate in the TENG-based sensors can improve the chemical selectivity and increase the chemical species to be detected.

\section{Acknowledgements}

We gratefully acknowledge the financial support from the Center for Advanced Soft Electronics funded by the Ministry of Education Science and Technology as a "Global Frontier Project" (CASE-2015M3A6A5072945) and the Korea Research Institute of Chemical Technology (KRICT). This work was also supported by the National Research Foundation of Korea (NRF) grant (no. 2013R1A3A2042196) funded by the Korean government. J.H.L. was supported by the Global PhD Fellowship program (2015H1A2A1033923) funded by the Ministry of Education, Korea.

\section{Conflict of interest}

The authors declare that they have no conflict of interest.

\section{Publisher's note}

Springer Nature remains neutral with regard to jurisdictional claims in published maps and institutional affiliations.

Received: 6 May 2019 Revised: 19 September 2019 Accepted: 6 October 2019.

Published online: 24 January 2020

\section{References}

1. Cao, X., Jie, Y., Wang, N. \& Wang, Z. L. Triboelectric nanogenerators driven self-powered electrochemical processes for energy and environmental science. Adv. Energy Mater. 6, 1600665 (2016).

2. Kwon, S. H. et al. Fabric active transducer stimulated by water motion for self powered wearable device. ACS Appl. Mater. Interfaces 8, 24579-24584 (2016).

3. Wang, S. H., Lin, L. \& Wang, Z. L. Triboelectric nanogenerators as self-powered active sensors. Nano Energy 11, 436-462 (2015).

4. Wu, C. S., Wang, A. C., Ding, W. B., Guo, H. Y. \& Wang, Z. L. Triboelectric nanogenerator: a foundation of the energy for the new era. Adv. Energy Mater. 9, 1802906 (2019).

5. Wang, Z. L. Triboelectric nanogenerators as new energy technology for selfpowered systems and as active mechanical and chemical sensors. ACS Nano 7, 9533-9557 (2013).

6. Niu, S. M. et al. Theoretical study of contact-mode triboelectric nanogenerators as an effective power source. Energy Environ. Sci. 6, 3576-3583 (2013).

7. Zhang, H. L. et al. Triboelectric nanogenerator built inside clothes for selfpowered glucose biosensors. Nano Energy 2, 1019-1024 (2013).

8. Yang, B. et al. A fully verified theoretical analysis of contact-mode triboelectric nanogenerators as a wearable power source. Adv. Energy Mater. 6, 1600505 (2016)
9. Niu, S. M. et al. Theory of sliding-mode triboelectric nanogenerators. Adv. Mater. 25, 6184-6193 (2013).

10. Zhu, G. et al. A shape-adaptive thin-film-based approach for $50 \%$ highefficiency energy generation through micro-grating sliding electrification. Adv. Mater. 26, 3788-3796 (2014).

11. Meng, B. et al. A transparent single-friction-surface triboelectric generator and self-powered touch sensor. Energy Environ. Sci. 6, 3235-3240 (2013).

12. Chen, S. W. et al. An ultrathin flexible single-electrode triboelectric-nanogenerator for mechanical energy harvesting and instantaneous force sensing. Adv. Energy Mater. 7, 1601255 (2017).

13. Zhou, T. et al. Woven structured triboelectric nanogenerator for wearable devices. ACS Appl. Mater. Interfaces 6, 14695-14701 (2014).

14. Zhao, Z. F. et al. Freestanding flag-type triboelectric nanogenerator for harvesting high-altitude wind energy from arbitrary directions. ACS Nano 10, 1780-1787 (2016).

15. Zheng, Q. et al. In vivo powering of pacemaker by breathing-driven implanted triboelectric nanogenerator. Adv. Mater. 26, 5851-5856 (2014).

16. Ko, Y. H., Lee, S. H., Leem, J. W. \& Yu, J. S. High transparency and triboelectric charge generation properties of nano-patterned PDMS. RSC Adv. 4, 10216-10220 (2014).

17. Fan, F. R. et al. Highly transparent and flexible triboelectric nanogenerators: performance improvements and fundamental mechanisms. J. Mater. Chem A 2, 13219-13225 (2014).

18. Kim, S. et al. Transparent flexible graphene triboelectric nanogenerators. Adv. Mater. 26, 3918-3925 (2014).

19. Zhang, X. S. et al. Frequency-multiplication high-output triboelectric nanogenerator for sustainably powering biomedical microsystems. Nano Lett. 13, 1168-1172 (2013).

20. Xi, F. B. et al. Universal power management strategy for triboelectric nanogenerator. Nano Energy 37, 168-176 (2017).

21. Zhao, Z. Z. et al. Ultrasensitive triboelectric nanogenerator for weak ambient energy with rational unipolar stacking structure and low-loss power management. Nano Energy 41, 351-358 (2017).

22. Luo, L. C., Bao, D. C., Yu, W. Q., Zhang, Z. H. \& Ren, T. L. A low input current and wide conversion ratio buck regulator with $75 \%$ efficiency for highvoltage triboelectric nanogenerators. Sci. Rep. 6, 19246 (2016).

23. Wang, Z. L. Triboelectric nanogenerators as new energy technology and selfpowered sensors - principles, problems and perspectives. Faraday Discuss. 176, 447-458 (2014).

24. Lee, K. Y. et al. Hydrophobic sponge structure-based triboelectric nanogenerator. Adv. Mater. 26, 5037-5042 (2014).

25. Zou, H. Y. et al. Quantifying the triboelectric series. Nat. Commun. 10, 1427 (2019).

26. Zhu, G. et al. Toward large-scale energy harvesting by a nanoparticleenhanced triboelectric nanogenerator. Nano Lett. 13, 847-853 (2013).

27. Jiang, $\mathrm{T}$. et al. Structural optimization of triboelectric nanogenerator for harvesting water wave energy. ACS Nano 9, 12562-12572 (2015).

28. Niu, S. M. \& Wang, Z. L. Theoretical systems of triboelectric nanogenerators. Nano Energy 14, 161-192 (2015).

29. Zi, Y. L. et al. Standards and figure-of-merits for quantifying the performance of triboelectric nanogenerators. Nat. Commun. 6, 8376 (2015).

30. Jang, D. et al. Force-assembled triboelectric nanogenerator with highhumidity-resistant electricity generation using hierarchical surface morphology. Nano Energy 20, 283-293 (2016).

31. Seol, M. L. et al. Nature-replicated nano-in-micro structures for triboelectric energy harvesting. Small 10, 3887-3894 (2014).

32. Bai, P. et al. Integrated multilayered triboelectric nanogenerator for harvesting biomechanical energy from human motions. ACS nano 7, 3713-3719 (2013).

33. Jeong, C. K. et al. Topographically-designed triboelectric nanogenerator via block copolymer self-assembly. Nano Lett. 14, 7031-7038 (2014).

34. Zhu, G. et al. Triboelectric-generator-driven pulse electrodeposition for micropatterning. Nano Lett. 12, 4960-4965 (2012).

35. Lin, Z. H. et al. Enhanced triboelectric nanogenerators and triboelectric nanosensor using chemically modified TiO2 nanomaterials. ACS Nano 7, 4554-4560 (2013).

36. Choi, H. J. et al. High-performance triboelectric nanogenerators with artifcially well-tailored interlocked interfaces. Nano Energy 27, 595-601 (2016).

37. Lee, J. H., Yu, I., Hyun, S., Kim, J. K. \& Jeong, U. Remarkable increase in triboelectrification by enhancing the conformable contact and adhesion energy with a film-covered pillar structure. Nano Energy 34, 233-241 (2017). 
38. Yoo, D. et al. Biomimetic anti-reflective triboelectric nanogenerator for concurrent harvesting of solar and raindrop energies. Nano Energy 57, 424-431 (2019).

39. Lee, J. W. \& Hwang, W. Theoretical study of micro/nano roughness effect on water-solid triboelectrification with experimental approach. Nano Energy $\mathbf{5 2}$, 315-322 (2018).

40. Nie, J. et al. Power generation from the interaction of a liquid droplet and a liquid membrane. Nat. Commun. 10, 2264 (2019).

41. Song, G. et al. Molecularly engineered surface triboelectric nanogenerator by self-assembled monolayers (mets). Chem. Mater. 27, 4749-4755 (2015).

42. Wang, S. H. et al. Maximum surface charge density for triboelectric nanogenerators achieved by ionized-air injection: Methodology and theoretical understanding. Adv. Mater. 26, 6720-6728 (2014).

43. Yun, B. K. et al. Base-treated polydimethylsiloxane surfaces as enhanced triboelectric nanogenerators. Nano Energy 15, 523-529 (2015).

44. Zhang, X. S. et al. High-performance triboelectric nanogenerator with enhanced energy density based on single-step fluorocarbon plasma treatment. Nano Energy 4, 123-131 (2014).

45. Li, H. Y. et al. Significant enhancement of triboelectric charge density by fluorinated surface modification in nanoscale for converting mechanical energy. Adv. Funct. Mater. 25, 5691-5697 (2015).

46. Byun, K. E. et al. Control of triboelectrification by engineering surface dipole and surface electronic state. ACS Appl. Mater. Interfaces 8, 18519-18525 (2016).

47. Wang, S. H. et al. Molecular surface functionalization to enhance the powe output of triboelectric nanogenerators. J. Mater. Chem. A 4, 3728-3734 (2016).

48. Ducrot, P. H., Dufour, I. \& Ayela, C. Optimization of PVDF-TrFE processing conditions for the fabrication of organic MEMS resonators. Sci. Rep. 6, 19426 (2016).

49. Lee, J. H. et al. Control of skin potential by triboelectrification with ferroelectric polymers. Adv. Mater. 27, 5553-5558 (2015)

50. Seung, W. et al. Boosting power-generating performance of triboelectric nanogenerators via artificial control of ferroelectric polarization and dielectric properties. Adv. Energy Mater. 7, 1600988 (2017).

51. Ryu, $\mathrm{H}$. et al. High-performance triboelectric nanogenerators based on solid polymer electrolytes with asymmetric pairing of ions. Adv. Energy Mater. $\mathbf{7}$ 1700289 (2017).

52. Kim, K. N. et al. Surface dipole enhanced instantaneous charge pair generation in triboelectric nanogenerator. Nano Energy 26, 360-370 (2016).

53. Lee, J. W. et al. Robust nanogenerators based on graft copolymers via control of dielectrics for remarkable output power enhancement. Sci. Adv. 3 e1602902 (2017)

54. Kim, D. W., Kim, S. W. \& Jeong, U. Lipids: source of static electricity of regenerative natural substances and nondestructive energy harvesting. Adv. Mater. 30, 1804949 (2018).

55. Meder, F. et al. Energy conversion at the cuticle of living plants. Adv. Funct. Mater. 28, 1806689 (2018).

56. Chen, Y. D. et al. Triboelectrification on natural rose petal for harvesting environmental mechanical energy. Nano Energy 50, 441-447 (2018).

57. Seol, M. et al. Triboelectric series of $2 \mathrm{D}$ layered materials. Adv. Mater. 30 1801210 (2018).

58. Singh, H. H. \& Khare, N. Improved performance of ferroelectric nanocomposite flexible film based triboelectric nanogenerator by controlling surface morphology, polarizability, and hydrophobicity. Energy 178, 765-771 (2019)

59. Soin, $\mathrm{N}$. et al. High performance triboelectric nanogenerators based on phase-inversion piezoelectric membranes of poly(vinylidene fluoride)-zinc stannate (PVDF-ZnSnO3) and polyamide-6 (PA6). Nano Energy 30, 470-480 (2016).

60. Shen, W. W., Mu, H. B., Zhang, G. J., Deng, J. B. \& Tu, D. M. Identification of electron and hole trap based on isothermal surface potential decay model. J. Appl. Phys. 113, 083706 (2013)

61. Li, J. Y., Zhou, F. S., Min, D. M., Li, S. T. \& Xia, R. The energy distribution of trapped charges in polymers based on isothermal surface potential decay model. IEEE Trans. Dielectr. Electr. Insul. 22, 1723-1732 (2015).

62. Takada, $T$. et al. Determination of charge-trapping sites in saturated and aromatic polymers by quantum chemical calculation. IEEE Trans. Dielectr. Electr. Insul. 22, 1240-1249 (2015).

63. Cui, N. Y. et al. Dynamic behavior of the triboelectric charges and structural optimization of the friction layer for a triboelectric nanogenerator. ACS Nano 10, 6131-6138 (2016)
64. Feng, Y. G. et al. A new protocol toward high output teng with polyimide as charge storage layer. Nano Energy 38, 467-476 (2017).

65. Kim, D. W., Lee, J. H., You, I., Kim, J. K. \& Jeong, U. Adding a stretchable deeptrap interlayer for high-performance stretchable triboelectric nanogenerators. Nano Energy 50, 192-200 (2018).

66. Park, H. W. et al. Electron blocking layer-based interfacial design for highlyenhanced triboelectric nanogenerators. Nano Energy 50, 9-15 (2018).

67. Park, D., Lee, S., Anh, C. V., Park, P. \& Nah, J. Role of a buried indium zinc oxide layer in the performance enhancement of triboelectric nanogenerators. Nano Energy 55, 501-505 (2019).

68. Wu, C. et al. Enhanced triboelectric nanogenerators based on MoS2 monolayer nanocomposites acting as electron-acceptor layers. ACS Nano $\mathbf{1 1}$ 8356-8363 (2017).

69. Wu, C., Kim, T. W. \& Choi, H. Y. Reduced graphene-oxide acting as electrontrapping sites in the friction layer for giant triboelectric enhancement. Nano Energy 32, 542-550 (2017).

70. Xiong, J. Q. et al. Skin-touch-actuated textile-based triboelectric nanogenerator with black phosphorus for durable biomechanical energy harvesting Nat. Commun. 9, 4280 (2018).

71. Cheon, S. et al. High-performance triboelectric nanogenerators based on electrospun polyvinylidene fluoride-silver nanowire composite nanofibers. Adv. Funct. Mater. 28, 1703778 (2018).

72. Wen, R. M., Guo, J. M., Yu, A. F., Zhai, J. Y. \& Wang, Z. L. Humidity-resistive triboelectric nanogenerator fabricated using metal organic framework composite. Adv. Funct. Mater. 29, 1807655 (2019)

73. Wen, R. M. et al. Remarkably enhanced triboelectric nanogenerator based on flexible and transparent monolayer titania nanocomposite. Nano Energy $\mathbf{5 0}$ 140-147 (2018).

74. Lin, Z. M. et al. Triboelectric nanogenerator enabled body sensor network for self-powered human heart-rate monitoring. ACS Nano 11, 8830-8837 (2017).

75. Seung, W. et al. Nanopatterned textile-based wearable triboelectric nanogenerator. ACS Nano 9, 3501-3509 (2015).

76. Zhang, L. et al. Lawn structured triboelectric nanogenerators for scavenging sweeping wind energy on rooftops. Adv. Mater. 28, 1650-1656 (2016).

77. $\mathrm{Xu}, \mathrm{S}$. et al. Stretchable batteries with self-similar serpentine interconnects and integrated wireless recharging systems. Nat. Commun. 4, 1543 (2013).

78. Yang, P. K. et al. A flexible, stretchable and shape-adaptive approach for versatile energy conversion and self-powered biomedical monitoring. Adv Mater. 27, 3817-3824 (2015).

79. Vosgueritchian, M., Lipomi, D. J. \& Bao, Z. A. Highly conductive and transparent PEDOT: PSS films with a fluorosurfactant for stretchable and flexible transparent electrodes. Adv. Funct. Mater. 22, 421-428 (2012).

80. Wang, C. Y., Zheng, W., Yue, Z. L., Too, C. O. \& Wallace, G. G. Buckled, stretchable polypyrrole electrodes for battery applications. Adv. Mater. 23, 3580-3584 (2011).

81. Hyun, D. C. et al. Ordered zigzag stripes of polymer gel/metal nanoparticle composites for highly stretchable conductive electrodes. Adv. Mater. 23, 2946-2950 (2011).

82. Amjadi, M., Kyung, K. U., Park, I. \& Sitti, M. Stretchable, skin-mountable, and wearable strain sensors and their potential applications: a review. Adv. Funct. Mater. 26, 1678-1698 (2016).

83. Matsuhisa, N. et al. Printable elastic conductors with a high conductivity for electronic textile applications. Nat. Commun. 6, 7461 (2015).

84. Moon, G. D. et al. Highly stretchable patterned gold electrodes made of Au nanosheets. Adv. Mater. 25, 2707-2712 (2013).

85. Yi, F. et al. Stretchable and waterproof self-charging power system for harvesting energy from diverse deformation and powering wearable electronics. ACS nano 10, 6519-6525 (2016).

86. Lai, Y. C. et al. Electric eel-skin-inspired mechanically durable and superstretchable nanogenerator for deformable power source and fully autonomous conformable electronic-skin applications. Adv. Mater. 28, 10024-10032 (2016).

87. Lim, G. H. et al. Fully stretchable and highly durable triboelectric nanogenerators based on gold-nanosheet electrodes for self-powered humanmotion detection. Nano Energy 42, 300-306 (2017).

88. Dickey, M. D. Stretchable and soft electronics using liquid metals. Adv. Mater. 29, 1606425 (2017).

89. Matsuzaki, R. \& Tabayashi, K. Highly stretchable, global, and distributed local strain sensing line using gainsn electrodes for wearable electronics. Adv. Funct. Mater. 25, 3806-3813 (2015). 
90. Yoon, J. et al. Design and fabrication of novel stretchable device arrays on a deformable polymer substrate with embedded liquid-metal interconnections. Adv. Mater. 26, 6580-6586 (2014).

91. Yang, Y. Q. et al. Liquid-metal-based super-stretchable and structuredesignable triboelectric nanogenerator for wearable electronics. ACS Nano 12, 2027-2034 (2018).

92. Yi, F. et al. A highly shape-adaptive, stretchable design based on conductive liquid for energy harvesting and self-powered biomechanical monitoring. Sci. Adv. 2, e1501624 (2016).

93. Shi, J. et al. A liquid PEDOT: PSS electrode-based stretchable triboelectric nanogenerator for a portable self-charging power source. Nanoscale $\mathbf{1 1}$ 7513-7519 (2019).

94. Sun, J. Y. et al. Highly stretchable and tough hydrogels. Nature 489, 133-136 (2012).

95. Keplinger, C. et al. Stretchable, transparent, ionic conductors. Science $\mathbf{3 4 1}$ 984-987 (2013).

96. Liu, T. et al. Triboelectric-nanogenerator-based soft energy-harvesting skin enabled by toughly bonded elastomer/hydrogel hybrids. ACS Nano $\mathbf{1 2}$ 2818-2826 (2018).

97. Parida, K. et al. Highly transparent, stretchable, and self-healing ionic-skin triboelectric nanogenerators for energy harvesting and touch applications. Adv. Mater. 29, 1702181 (2017).

98. Lee, Y., Cha, S. H., Kim, Y. W., Choi, D. \& Sun, J. Y. Transparent and attachable ionic communicators based on self-cleanable triboelectric nanogenerators. Nat. Commun. 9, 1804 (2018).

99. Niu, S., Wang, X., Yi, F., Zhou, Y. S. \& Wang, Z. L. A universal self-charging system driven by random biomechanical energy for sustainable operation of mobile electronics. Nat. Commun. 6, 8975 (2015).

100. Nan, X. et al. Highly efficient storage of pulse energy produced by triboelectric nanogenerator in Li3V2(PO4)3/C cathode Li-ion batteries. ACS Appl. Mater. Interfaces 8, 862-870 (2016).

101. Wang, S. H. et al. Motion charged battery as sustainable flexible-power-unit. ACS Nano 7, 11263-11271 (2013).

102. Zhang, X. L. et al. Lithium-ion batteries: charged by triboelectric nanogenerators with pulsed output based on the enhanced cycling stability. ACS Appl. Mater. Interfaces 10, 8676-8684 (2018).

103. Liu, X., Zhao, K., Wang, Z. L. \& Yang, Y. Unity convoluted design of solid Li-ion battery and triboelectric nanogenerator for self-powered wearable electronics. Adv. Energy Mater. 7, 1701629 (2017).

104. Xue, X. Y., Wang, S. H., Guo, W. X., Zhang, Y. \& Wang, Z. L. Hybridizing energy conversion and storage in a mechanical-to-electrochemical process for selfcharging power cell. Nano Lett. 12, 5048-5054 (2012)

105. Gao, T. T., Zhao, K., Liu, X. \& Yang, Y. Implanting a solid Li-ion battery into a triboelectric nanogenerator for simultaneously scavenging and storing wind energy. Nano Energy 41, 210-216 (2017).

106. $\mathrm{Pu}, \mathrm{X}$. et al. Efficient charging of Li-ion batteries with pulsed output current of triboelectric nanogenerators. Adv. Sci. 3, 1500255 (2016).

107. Li, C. I. et al. Van der waal epitaxy of flexible and transparent VO2 film on muscovite. Chem. Mater. 28, 3914-3919 (2016).

108. Bakaul, S. R. et al. High speed epitaxial perovskite memory on flexible substrates. Adv. Mater. 29, 1605699 (2017).

109. Liang, Z. et al. All-inorganic flexible embedded thin-film capacitors for dielectric energy storage with high performance. ACS Appl. Mater. Interfaces 11, 5247-5255 (2019)

110. Chung, J. et al. Capacitor-integrated triboelectric nanogenerator based on metal-metal contact for current amplification. Adv. Energy Mater. 8, 1703024 (2018).

111. Kim, B. J. et al. Water-gel for gating graphene transistors. Nano Lett. 14, 2610-2616 (2014).

112. Cho, J. H. et al. Printable ion-gel gate dielectrics for low-voltage polymer thinfilm transistors on plastic. Nat. Mater. 7, 900-906 (2008)

113. Guo, H. Y. et al. All-in-one shape-adaptive self-charging power package for wearable electronics. ACS Nano 10, 10580-10588 (2016).

114. Song, Y. et al. Integrated self-charging power unit with flexible supercapacitor and triboelectric nanogenerator. J. Mater. Chem. A 4, 14298-14306 (2016).

115. Wang, J. et al. A flexible fiber-based supercapacitor-triboelectricnanogenerator power system for wearable electronics. Adv. Mater. 27, 4830-4836 (2015).

116. Yuan, C. Z., Chen, L., Gao, B., Su, L. H. \& Zhang, X. G. Synthesis and utilization of $\mathrm{RuO} 2 \times \mathrm{H} 2 \mathrm{O}$ nanodots well dispersed on poly(sodium 4-styrene sulfonate) functionalized multi-walled carbon nanotubes for supercapacitors. J. Mater Chem. 19, 246-252 (2009).

117. Pico, F. et al. Understanding $\mathrm{RuO} 2 \times \mathrm{H} 2 \mathrm{O} /$ carbon nanofibre composites as supercapacitor electrodes. J. Power Sources 176, 417-425 (2008).

118. Lee, J. H. et al. Output voltage modulation in triboelectric nanogenerator by printed ion gel capacitors. Nano Energy 54, 367-374 (2018)

119. Kong, D. et al. Capacitance characterization of elastomeric dielectrics for applications in intrinsically stretchable thin film transistors. Adv. Funct. Mater. 26, 4680-4686 (2016).

120. Hammock, M. L., Chortos, A., Tee, B. C. K., Tok, J. B. H. \& Bao, Z. A. 25th anniversary article: the evolution of electronic skin (e-skin): a brief history, design considerations, and recent progress. Adv. Mater. 25, 5997-6037 (2013).

121. Wang, X. D. et al. Recent progress in electronic skin. Adv. Sci. 2, 1500169 (2015).

122. Hua, Q. L. et al. Skin-inspired highly stretchable and conformable matrix networks for multifunctional sensing. Nat. Commun. 9, 244 (2018).

123. Zhu, G. et al. Self-powered, ultrasensitive, flexible tactile sensors based on contact electrification. Nano Lett. 14, 3208-3213 (2014).

124. Lin, L. et al. Triboelectric active sensor array for self-powered static and dynamic pressure detection and tactile imaging. ACS Nano 7, 8266-8274 (2013).

125. Ren, Z. et al. Fully elastic and metal-free tactile sensors for detecting both normal and tangential forces based on triboelectric nanogenerators. Adv Funct. Mater. 28, 1802989 (2018).

126. $\mathrm{Pu}, \mathrm{X}$. J. et al. Eye motion triggered self-powered mechnosensational communication system using triboelectric nanogenerator. Sci. Adv. 3, e1700694 (2017).

127. Yang, J. et al. Eardrum-inspired active sensors for self-powered cardiovascular system characterization and throat-attached anti-interference voice recognition. Adv. Mater. 27, 1316-1326 (2015).

128. Jin, L. M., Tao, J., Bao, R. R., Sun, L. \& Pan, C. F. Self-powered real-time movement monitoring sensor using triboelectric nanogenerator technology. Sci. Rep. 7, 10521 (2017).

129. Lu, C. X. et al. A stretchable, flexible triboelectric nanogenerator for selfpowered real-time motion monitoring. Adv. Mater. Technol. 3, 1800021 (2018).

130. Chen, X. et al. On-skin triboelectric nanogenerator and self-powered sensor with ultrathin thickness and high stretchability. Small 13, 1702929 (2017).

131. $\mathrm{Pu}, \mathrm{X}$. et al. Ultrastretchable, transparent triboelectric nanogenerator as electronic skin for biomechanical energy harvesting and tactile sensing. Sci. Adv. 3, e1700015 (2017)

132. Zhao, G. R. et al. Transparent and stretchable triboelectric nanogenerator for self-powered tactile sensing. Nano Energy 59, 302-310 (2019).

133. Xu, W. et al. Environmentally friendly hydrogel-based triboelectric nanogenerators for versatile energy harvesting and self-powered sensors. Adv. Energy Mater. 7, 1601529 (2017).

134. Lee, J. et al. Conductive fiber-based ultrasensitive textile pressure sensor for wearable electronics. Adv. Mater. 27, 2433-2439 (2015).

135. Song, J. H. et al. Surface-embedded stretchable electrodes by direct printing and their uses to fabricate ultrathin vibration sensors and circuits for 3D structures. Adv. Mater. 29, 1702625 (2017).

136. Lee, W. J. et al. Conductive magnetic-patchy colloidal microparticles for a high performance pressure sensor. Chem. Commun. 52, 12334-12337 (2016).

137. You, I. et al. E-skin tactile sensor matrix pixelated by position-registered conductive microparticles creating pressure-sensitive selectors. Adv. Funct. Mater. 28, 1801858 (2018).

138. Wang, P. H. et al. Complementary electromagnetic-triboelectric active sensor for detecting multiple mechanical triggering. Adv. Funct. Mater. 28, 1705808 (2018).

139. Li, T. et al. From dual-mode triboelectric nanogenerator to smart tactile sensor: a multiplexing design. ACS Nano 11, 3950-3956 (2017).

140. Choi, W. et al. Stretchable triboelectric multimodal tactile interface simultaneously recognizing various dynamic body motions. Nano Energy $\mathbf{5 6}$ 347-356 (2019).

141. Lin, Z. H. et al. A self-powered triboelectric nanosensor for mercury ion detection. Angew. Chem. Int. Ed. 52, 5065-5069 (2013).

142. Li, Z. L. et al. Beta-cyclodextrin enhanced triboelectrification for self-powered phenol detection and electrochemical degradation. Energy Environ. Sci. 8 887-896 (2015).

143. Choi, D. et al. A smart pipet tip: triboelectricity and thermoelectricity assisted in situ evaluation of electrolyte concentration. Nano Energy 38, 419-427 (2017). 
144. Jeon, S. B., Seol, M. L., Kim, D., Park, S. J. \& Choi, Y. K. Self-powered ion concentration sensor with triboelectricity from liquid-solid contact electrification. Adv. Electron. Mater. 2, 1600006 (2016).

145. Cui, S. W. et al. Self-powered ammonia nanosensor based on the integration of the gas sensor and triboelectric nanogenerator. Nano Energy 49, 31-39 (2018).

146. Virii, S., Huang, J. X., Kaner, R. B. \& Weiller, B. H. Polyaniline nanofiber gas sensors: examination of response mechanisms. Nano Lett. 4, 491-496 (2004).

147. Uddin, A. S. M. I. \& Chung, G. S. A self-powered active hydrogen gas sensor with fast response at room temperature based on triboelectric effect. Sens. Actuat. B-Chem. 231, 601-608 (2016)

148. Kim, J. H., Chun, J., Kim, J. W., Choi, W. J. \& Baik, J. M. Self-powered, roomtemperature electronic nose based on triboelectrification and heterogeneous catalytic reaction. Adv. Funct. Mater. 25, 7049-7055 (2015).

149. Xue, X. Y., Fu, Y. M., Wang, Q., Xing, L. L. \& Zhang, Y. Outputting olfactory bionic electric impulse by PANI/PTFE/PANI sandwich nanostructures and their application as flexible, smelling electronic skin. Adv. Funct. Mater. 26 3128-3138 (2016).

150. Ha, M., Park, J., Lee, Y. \& Ko, H. Triboelectric generators and sensors for selfpowered wearable electronics. ACS Nano 9, 3421-3427 (2015).

151. Henniker, J. Triboelectricity in polymers. Nature 196, 474-474 (1962).

152. Davies, D. K. Charge generation on dielectric surfaces. J. Phys. D Appl. Phys. 2, 1533-1537 (1969).

153. Apodaca, M. M., Wesson, P. J., Bishop, K. J. M., Ratner, M. A. \& Grzybowski, B. A. Contact electrification between identical materials. Angew. Chem. Int. Ed. 49, 946-949 (2010).

154. Lin, S. Q. et al. Electron transfer in nanoscale contact electrification: effect of temperature in the metal-dielectric case. Adv. Mater. 31, 1808197 (2019).

155. Sakaguchi, M., Makino, M., Ohura, T. \& Iwata, T. Contact electrification of polymers due to electron transfer among mechano anions, mechano cations and mechano radicals. J. Electrostat. 72, 412-416 (2014).

156. Diaz, A. F. \& Guay, J. Contact charging of organic materials - ion vs electrontransfer. IBM J. Res. Dev. 37, 249-259 (1993).

157. Choi, Y. S., Jing, Q. S., Datta, A., Boughey, C. \& Kar-Narayan, S. A triboelectric generator based on self-poled nylon-11 nanowires fabricated by gas-flow assisted template wetting. Energy Environ. Sci. 10, 2180-2189 (2017).
158. Johnston, I. D., McCluskey, D. K., Tan, C. K. L. \& Tracey, M. C. Mechanical characterization of bulk sylgard 184 for microfluidics and microengineering. J. Micromech. Microeng. 24, 035017 (2014).

159. Chun, J. S. et al. Boosted output performance of triboelectric nanogenerator via electric double layer effect. Nat. Commun. 7, 12985 (2016).

160. Wang, X. et al. Self-powered high-resolution and pressure-sensitive triboelectric sensor matrix for real-time tactile mapping. Adv. Mater. 28, 2896-2903 (2016).

161. Ghaffarinejad, A. et al. A conditioning circuit with exponential enhancement of output energy for triboelectric nanogenerator. Nano Energy 51, 173-184 (2018).

162. Park, J. et al. Tactile-direction-sensitive and stretchable electronic skins based on human-skin-inspired interlocked microstructures. ACS Nano $\mathbf{8}$, 12020-12029 (2014).

163. Park, S. et al. Stretchable energy-harvesting tactile electronic skin capable of differentiating multiple mechanical stimuli modes. Adv. Mater. 26, 7324-7332 (2014).

164. Zheng, Q. et al. Biodegradable triboelectric nanogenerator as a life-time designed implantable power source. Sci. Adv. 2, e1501478 (2016).

165. Ma, Y. et al. Self-powered, one-stop, and multifunctional implantable triboelectric active sensor for real-time biomedical monitoring. Nano Lett. $\mathbf{1 6}$ 6042-6051 (2016).

166. Ouyang, $\mathrm{H}$. et al. Self-powered pulse sensor for antidiastole of cardiovascular disease. Adv. Mater. 29, 1703456 (2017).

167. Wang, S. et al. A facile respiration-driven triboelectric nanogenerator for multifunctional respiratory monitoring. Nano Energy 58, 312-321 (2019).

168. Lee, S. et al. Mechano-neuromodulation of autonomic pelvic nerve for underactive bladder: a triboelectric neurostimulator integrated with flexible neural clip interface. Nano Energy 60, 449-456 (2019).

169. Khan, U., Kim, T. H., Ryu, H., Seung, W. \& Kim, S. W. Graphene tribotronics for electronic skin and touch screen applications. Adv. Mater. 29, 1603544 (2017).

170. Arshak, K. \& Gaidan, I. Development of a novel gas sensor based on oxide thick films. Mater. Sci. Eng. B 118, 44-49 (2005).

171. Bai, H. \& Shi, G. Q. Gas sensors based on conducting polymers. Sensors 7, 267-307 (2007). 